\title{
Essential Role of Cyclophilin A for Hepatitis C Virus Replication and Virus Production and Possible Link to Polyprotein Cleavage Kinetics
}

\author{
Artur Kaul', Sarah Stauffer ${ }^{19}$, Carola Berger $^{19}$, Thomas Pertel ${ }^{2}$, Jennifer Schmitt ${ }^{1}$, Stephanie Kallis ${ }^{1}$, \\ Margarita Zayas Lopez ${ }^{1}$, Volker Lohmann ${ }^{1}$, Jeremy Luban ${ }^{2}$, Ralf Bartenschlager ${ }^{1 *}$
}

1 Department of Molecular Virology, University of Heidelberg, Heidelberg, Germany, 2 Department of Microbiology and Molecular Medicine, University of Geneva, Geneva, Switzerland

\begin{abstract}
Viruses are obligate intracellular parasites and therefore their replication completely depends on host cell factors. In case of the hepatitis C virus (HCV), a positive-strand RNA virus that in the majority of infections establishes persistence, cyclophilins are considered to play an important role in RNA replication. Subsequent to the observation that cyclosporines, known to sequester cyclophilins by direct binding, profoundly block HCV replication in cultured human hepatoma cells, conflicting results were obtained as to the particular cyclophilin (Суp) required for viral RNA replication and the underlying possible mode of action. By using a set of cell lines with stable knock-down of CypA or CypB, we demonstrate in the present work that replication of subgenomic HCV replicons of different genotypes is reduced by CypA depletion up to 1,000-fold whereas knock-down of СypB had no effect. Inhibition of replication was rescued by over-expression of wild type CypA, but not by a mutant lacking isomerase activity. Replication of JFH1-derived full length genomes was even more sensitive to CypA depletion as compared to subgenomic replicons and virus production was completely blocked. These results argue that CypA may target an additional viral factor outside of the minimal replicase contributing to RNA amplification and assembly, presumably nonstructural protein 2. By selecting for resistance against the cyclosporine analogue DEBIO-025 that targets CypA in a dose-dependent manner, we identified two mutations (V2440A and V2440L) close to the cleavage site between nonstructural protein $5 \mathrm{~A}$ and the RNA-dependent RNA polymerase in nonstructural protein 5B that slow down cleavage kinetics at this site and reduce CypA dependence of viral replication. Further amino acid substitutions at the same cleavage site accelerating processing increase CypA dependence. Our results thus identify an unexpected correlation between HCV polyprotein processing and CypA dependence of HCV replication.
\end{abstract}

\footnotetext{
Citation: Kaul A, Stauffer S, Berger C, Pertel T, Schmitt J, et al. (2009) Essential Role of Cyclophilin A for Hepatitis C Virus Replication and Virus Production and Possible Link to Polyprotein Cleavage Kinetics. PLoS Pathog 5(8): e1000546. doi:10.1371/journal.ppat.1000546

Editor: Charles M Rice, The Rockefeller University, United States of America

Received March 17, 2009; Accepted July 17, 2009; Published August 14, 2009

Copyright: $\odot 2009$ Kaul et al. This is an open-access article distributed under the terms of the Creative Commons Attribution License, which permits unrestricted use, distribution, and reproduction in any medium, provided the original author and source are credited.

Funding: This work was supported by grants from the Sonderforschungsbereich 638 (Teilprojekt A5 to R.B.), the Virgil network (EU grant LSHM-CT-2004-503359; to R.B.) and the National Institutes of Health (USA) grant RO1Al36199 as well as the Swiss National Science Foundation grant 3100A0-113558, both to J.L. The funders had no role in study design, data collection and analysis, decision to publish, or preparation of the manuscript.

Competing Interests: The authors have declared that no competing interests exist.

* E-mail: Ralf_Bartenschlager@med.uni-heidelberg.de

9 These authors contributed equally to this work.
}

\section{Introduction}

The hepatitis $\mathrm{C}$ virus (HCV) is a hepatotropic virus that has a high propensity to establish persistence. At present, more than 170 million people suffer from chronic hepatitis C [1]. Current therapy of this disease is based on the combination of pegylated interferonalpha and ribavirin. However, sustained viral response rates are not satisfying and side-effects associated with this therapy are high. Thus, the development of alternative and more effective strategies to counteract $\mathrm{HCV}$ infection are of great importance. One promising drug showing potent anti HCV function is cyclosporine A (CsA). The cyclic undecapeptide CsA is a secondary metabolite of the fungus Tolypocladium inflatum and was originally discovered as powerful immunosuppressive drug [2]. The immunosuppressive properties of CsA are due to its ability to block the phosphatase Calcineurin in activated T cells [3] by binding with high affinity to cyclophilins (CyPs) [4]. Because of the non-immunosuppressive properties combined with profound antiviral activity, CsA derivatives such as DEBIO-025 [5], NIM811 [6], and SCY-635 are more likely to be used as anti HCV agents. Unlike CsA, these molecules binds to GyPs but do not display calcineurin inhibition.

As a member of the Flaviviridae family, $\mathrm{HCV}$ has a positive strand RNA genome encoding a single polyprotein that is cleaved by cellular and viral proteases into 10 different proteins (reviewed in $[7,8])$. The structural proteins core, envelope protein 1 (E1) and $\mathrm{E} 2$ reside in the $\mathrm{N}$-terminal region of the polyprotein and they are the major constituents of the virus particle. Virus assembly and release requires $\mathrm{p} 7$, a presumed viroporin [9-11] and nonstructural protein 2 (NS2) that contains a complex N-terminal transmembrane domain and a $\mathrm{C}$-terminal protease domain responsible for cleavage between NS2 and NS3 [12,13]. The latter is composed of two domains, an N-terminal protease domain which is activated via the NS4A cofactor and a C-terminal helicase domain. NS4B most likely plays a major role in the induction of membrane alterations that are required for the assembly of viral replication complexes. NS5A is an RNA binding protein required 


\section{Author Summary}

Owing to limited genetic information, viruses have to exploit host cells to achieve efficient production of virus progeny. Host cell factors and pathways therefore play an important role for virus replication and thus represent a possible target for antiviral therapy. In case of the hepatitis $C$ virus (HCV), an RNA virus infecting liver cells and causing chronic liver disease, host cell cyclophilins were shown to play an important role in replication. Pharmacological inhibition of cyclophilins, which are catalysts of protein folding, causes profound inhibition of HCV replication, but neither the underlying mechanism by which cyclophilins contribute to viral replication, nor the exact nature of the cyclophilin are known. In this study we demonstrate that $\mathrm{HCV}$ replication and presumably also virus particle assembly requires cyclophilin $A(C y p A)$, which can be blocked by the cyclosporine analogue DEBIO-025. We identify mutations affecting proteolytic cleavage of the viral polyprotein that render HCV replication less dependent on CypA and thus cause DEBIO-025 resistance. Studies with additional mutants reveal a correlation between polyprotein cleavage kinetics and CypA dependence. Our results support a model by which CypA activates the viral replicase in a manner that depends on the kinetics with which the viral polyprotein is cleaved.

for replication and virus assembly [14-16] and NS5B is the RNAdependent RNA polymerase ( $\mathrm{RdRp})$. Cleavage of the polyprotein in the NS3 to NS5B region is mediated by the NS3/4A protease complex. Processing at the NS3-4A site is a rapid intramolecular reaction. Subsequent cleavages take place intermolecularly in the following preferred, but not obligatory order: NS5A-B, NS4A-B, NS4B-5A [17,18]. Replication of HCV occurs in the cytoplasm of infected cells in distinct virus-induced compartments designated the membranous web [19]. It is a complex membrane network composed of an accumulation of membranous vesicles of various sizes and interspersed lipid droplets which are the presumed sites of HCV assembly [16,20-22]. It is thought that core and NS5A are key players in mediating transfer of viral proteins and RNA from viral replication complexes to lipid droplets to trigger assembly $[16,21,22]$.

Our knowledge about cellular proteins required for HCV RNA replication and virus assembly is scarce, but recent studies suggest that cyclophilins (Cyp) play an important role. Cyps are molecular chaperones catalyzing the cis-trans isomerization of proline residues and hence are called peptidyl-prolyl cis-trans-isomerases (PPIases). Up to now 16 Cyp members have been identified with 7 major members found in humans (CypA-E, Cyp40, CypNK; [23]). Cyps share a common domain, but other than their PPIase activity differ in subcellular distribution and function. Conflicting data exist as to which Cyp is required for HCV replication. Watashi and coworkers reported that $\mathrm{CypB}$ is important for viral replication and they observed a direct binding of CypB to NS5B which results in enhanced RNA binding and thus increased RNA polymerase activity [24]. Others reported that CypA or CypA, B and $\mathrm{C}$ are required for HCV replication [25,26]. For both CypA [26] and CypB $[24,27,28]$ direct interactions with NS5B were reported arguing for a direct involvement of cyclophilins in $\mathrm{HCV}$ RNA replication. However, the underlying mechanism is not known.

We report here that an enzymatically active CypA is essential for HCV replication and describe an interrelation between CypA requirement for $\mathrm{HCV}$ replication and processing kinetics at the NS5A-B site.

\section{Results}

CypA but not CypB is essential for HCV replication and virus production

Contrary opinions about which type of Cyp is essential for HCV prevail in current literature $[24,26]$. To clarify these contradictions we established a panel of cell lines derived from the highly permissive Huh-7 subclones Huh7.5 and Huh7-Lunet by retroviral transduction of shRNAs targeting the $3^{\prime}$ non-coding region of CypA or CypB (Fig. 1). Cell pools transduced with the retroviral vector expressing an unrelated shRNA were generated as a control. Transduced shRNAs lead to a profound and stable reduction of the expression of CypA or CypB in the respective cell pools as detected by Western blot (Fig. 1A) and immunofluorescence analysis (Fig. 1B-C).

To determine the impact of reduced CypA or CypB expression on HCV RNA replication, cells were transfected with a subgenomic JFHl luciferase reporter replicon RNA (sgNS3/ JFH1-Luc; Fig. 2A, upper panel). Cells were lysed 4, 24, 48 and $72 \mathrm{~h}$ after transfection and replication was scored by measuring luciferase activity in cell lysates. Luciferase activity was normalized to the $4 \mathrm{~h}$ value, reflecting transfection efficiency. Replication was not affected by CypB knock-down and was comparable between naïve and vector control-transduced Huh7-Lunet or Huh7.5 cells (Fig. 2A, left and right panel, respectively). However, when we transfected the same replicon into cells with the stable CypA knock-down luciferase activity was reduced more than 100-fold at the early time point (24 h post transfection) in case of Huh7-Lunet cells and about 50 -fold in case of Huh7.5 cells. Only at the latest time point $(72 \mathrm{~h}$ post transfection) luciferase activities were comparable in all transfected cell lines. This kinetic is due to the fact that replication of JFH1 is limited by the host cell [29]. For this reason RNA replication phenotypes are often only detectable at early time points after transfection before host cell factor(s) (other than CypA) become limiting and thus restrict $\mathrm{HCV}$ replication in naïve cells. Therefore, HCV replication in cells with the CypA knock-down can 'catch up' until it reaches wild type level [30]. By using the luciferase values (which because of the very short half life of luciferase are ideally suited to determine replication kinetics) we calculated the apparent doubling time of HCV RNA during the exponential phase in the various cell lines. We found that apparent doubling time in naive and control cells were in the range of $3.7 \mathrm{~h}$ whereas apparent doubling time was 11.4 h in Huh7-Lunet cells with stable CypA knock-down. In case of Huh7.5-derived cell lines apparent doubling times were $3.6 \mathrm{~h}$ and $7.4 \mathrm{~h}$ for control and CypA knock-down cells, respectively. Thus results obtained with both cell lines were remarkably similar arguing against a cell line-specific phenotype.

Given the controversial discussion on genotypic differences with respect to Cyp-dependence of HCV replication, we also determined the impact of CypA and CypB knock-down on replicons derived from the genotype $1 \mathrm{~b}$ isolate Conl. As shown in Fig. 2B, replication of a highly adapted Conl-derived replicon (Con/ET; [31]) was completely blocked in Lunet cells with CypA knock-down as deduced from the comparable drop of luciferase expression in cells transfected with this replicon or the replication defective NS5B active site mutant (Con/GND). In contrast, replication of Con 1/ET was completely unaffected by knock-down of CypB expression. In case of Huh7.5 derived cell lines that support Conl RNA replication to a much lower extent, we also observed a complete inhibition of replication in CypA knock-down cells, but in some experiments a slight inhibition in cells with a CypB knock-down was found as well (Fig. 2B, right panel). The latter was however, at the limit of statistical significance (Mann-Whitney-Test). 
A

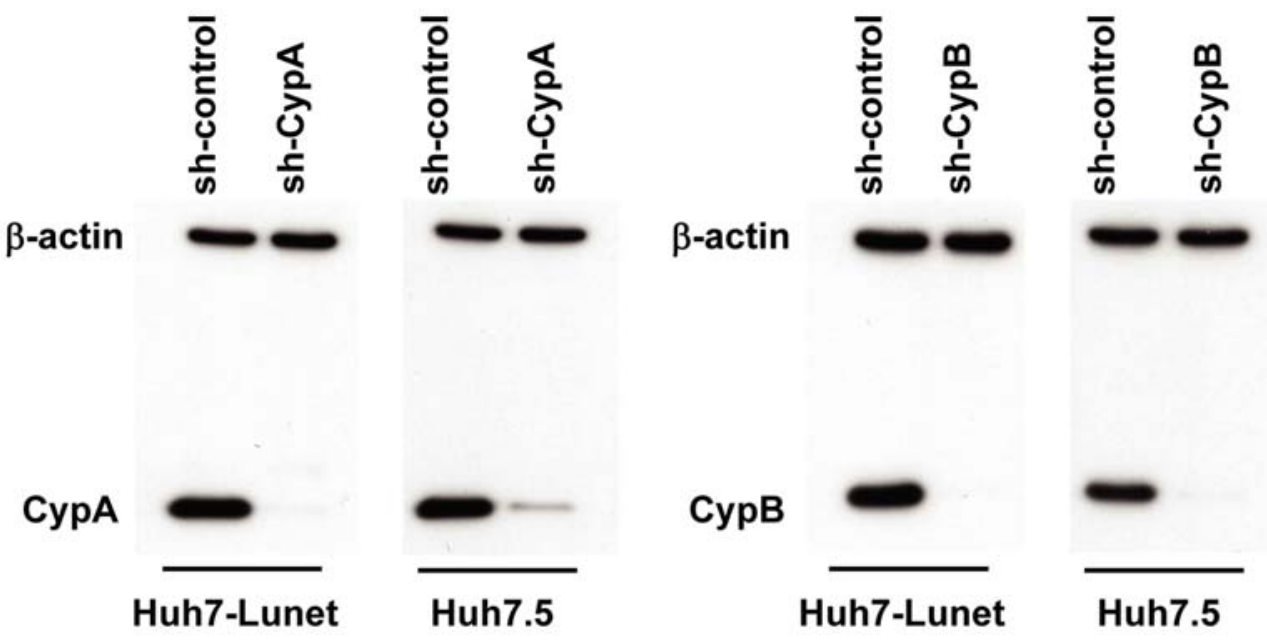

B Huh7-Lunet

Huh7.5
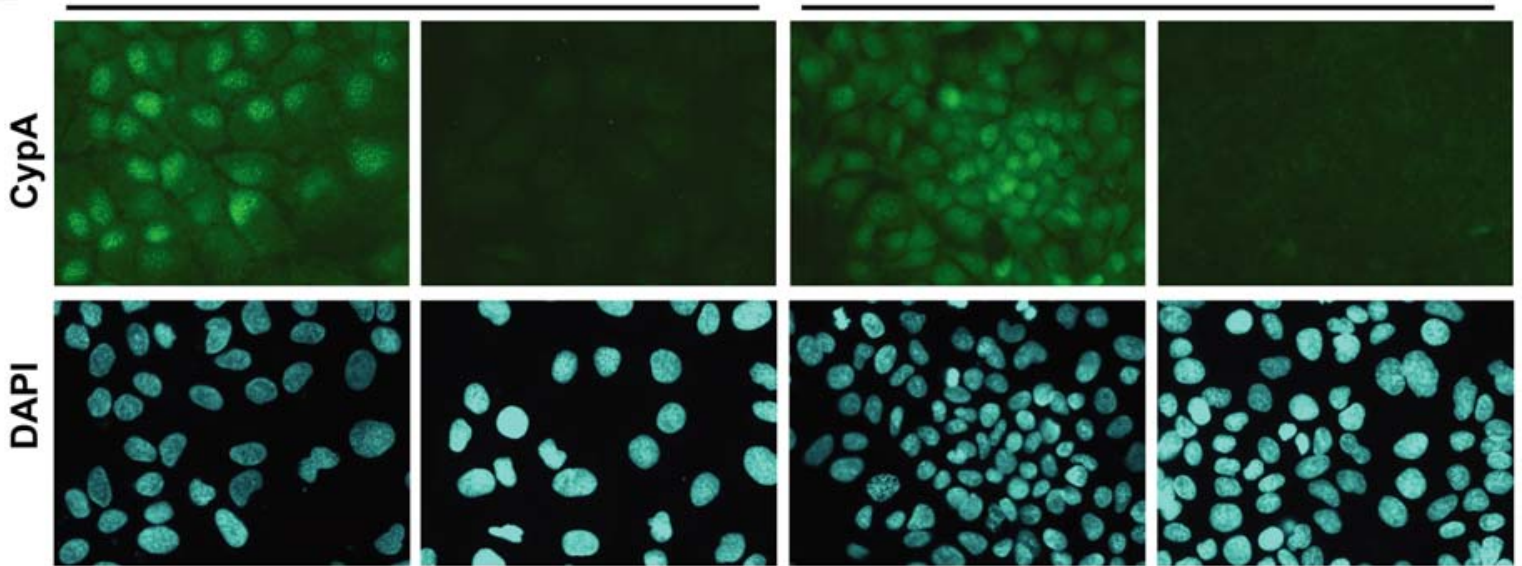

sh-control
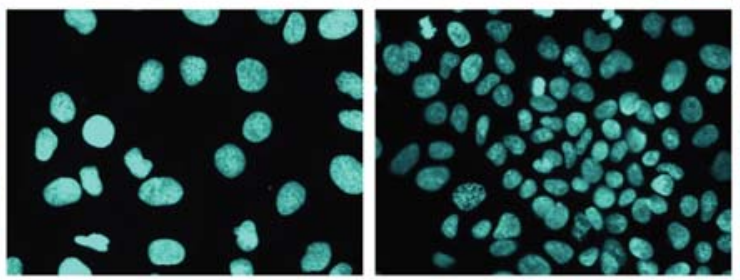

sh-control

sh-CурA

C

Huh7-Lunet
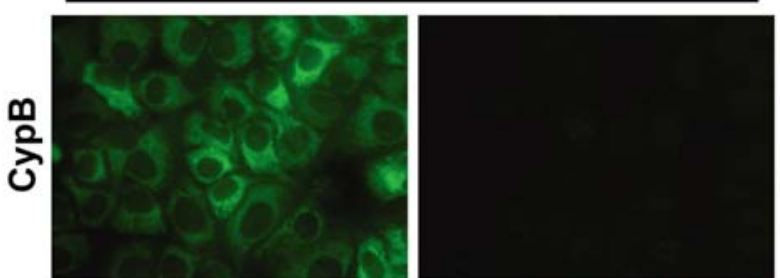

Huh7.5

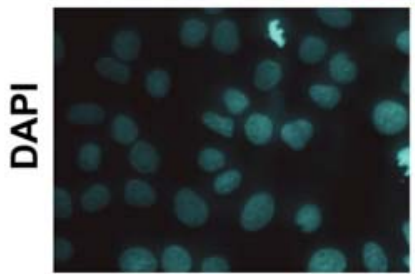

sh-control

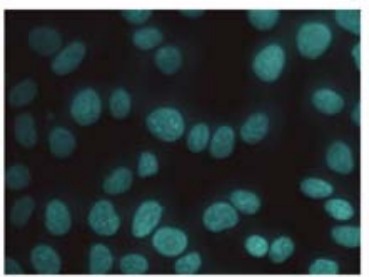

sh-CypB
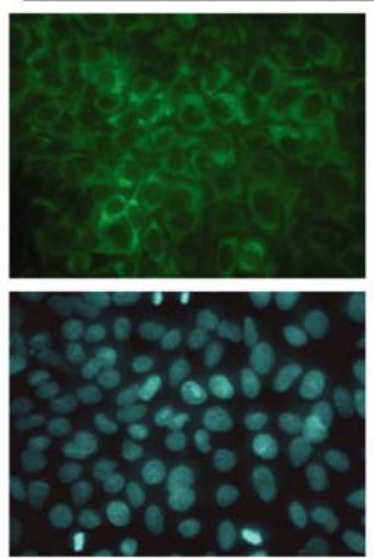

sh-control

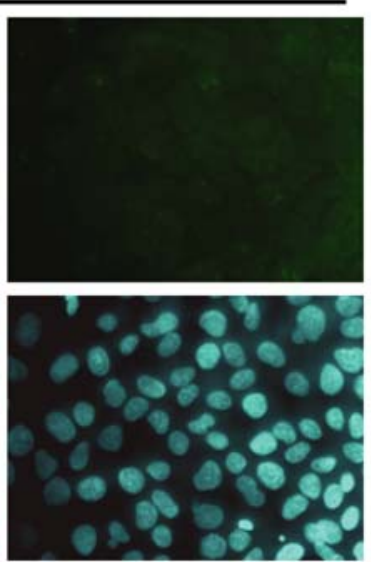

sh-CypB

Figure 1. Major role of CypA for HCV replication. (A-C) Stable knock-down of CypA (left panel) or CypB (right panel) in Huh7.5 and Huh7-Lunet cells was achieved by retroviral transduction of shRNAs targeting the $3^{\prime}$ NTR of either mRNA. As a reference, cells were transduced with the retroviral vector encoding an unrelated shRNA sequence (sh-control). (A) Knock-down efficiency was determined by western blot with a CypA or a CypB specific antibody. Beta-actin was used as loading control. (B, C) Detection of CypA (B) and CypB (C) knock-down in Huh7-Lunet and Huh7.5 cells via indirect immunofluorescence analysis. Cells were seeded onto glass cover slips and fixed with methanol $72 \mathrm{~h}$ later. Immunostaining was performed with CypA or CypB specific antibodies.

doi:10.1371/journal.ppat.1000546.g001 
A

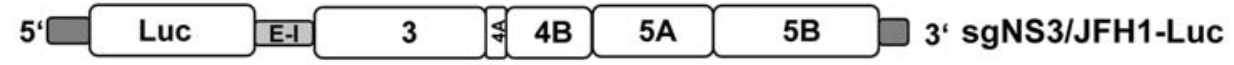

\section{naive $-\triangle$ sh-CypA $-\square$-sh-control - -sh-CypB}
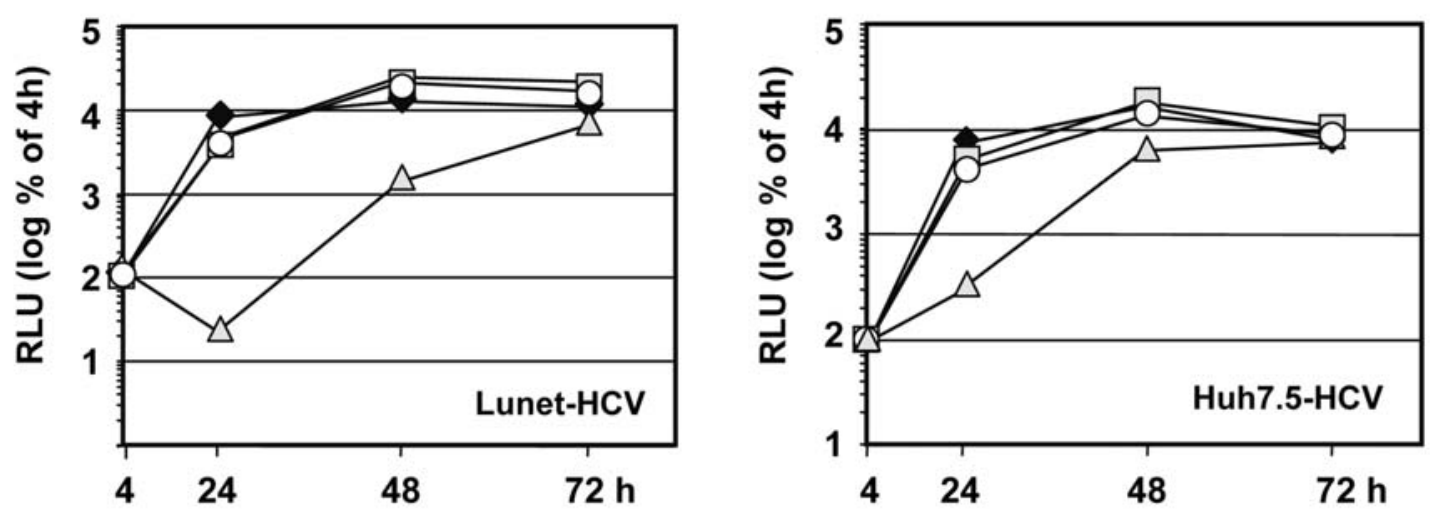

B

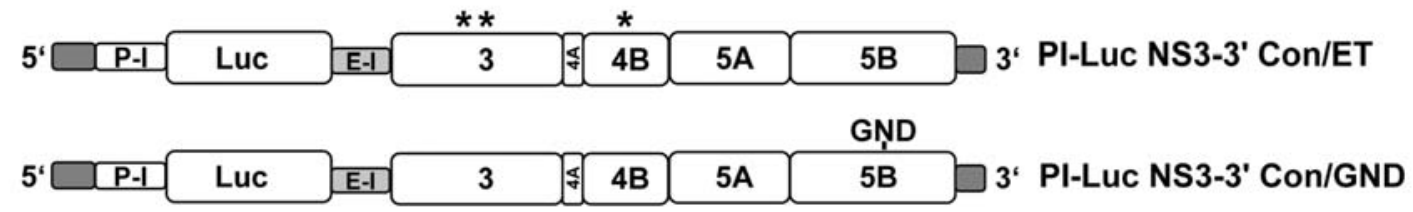

naive/ET $\triangle$ sh-CypA/ET $\square$-sh-control/ET $-O$ - sh-CypB/ET $\_$sh-CypA/GND
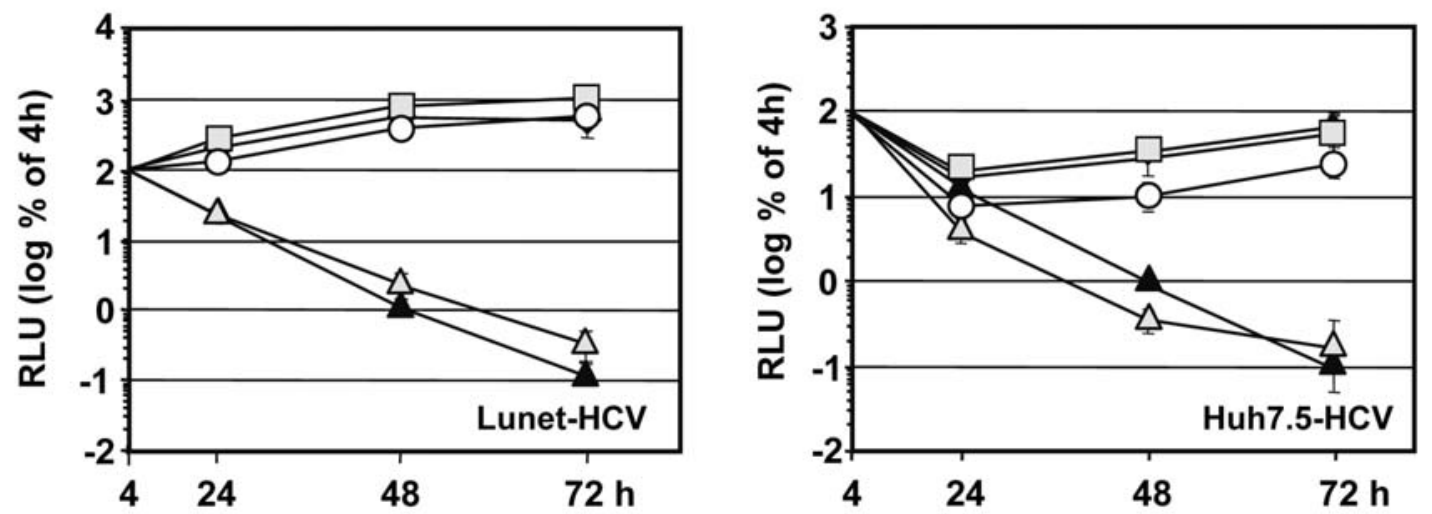

Figure 2. Impact of stable CypA or CypB knock-down on RNA replication of genotype 2a and 1b replicons. (A) Huh7-Lunet cells (left panel) and Huh7.5 cells (right panel) either stably transduced with the unrelated shRNA control (sh-control) or a vector encoding the CypA- or CypBspecific shRNA or naive cells were transfected with a subgenomic luciferase reporter replicon derived from the HCV isolate JFH1 (sgNS3/JFH1-Luc, shown at the top). Cells were lysed at given time points after transfection, and luciferase activity (expressed in relative light units, RLU) in cell lysates was determined. Values are normalized to the $4 \mathrm{~h}$ value that reflects transfection efficiency and that was set to $100 \%$. Means and standard deviations of a representative experiment are shown. Luc, firefly luciferase; sg, subgenomic replicon. (B) Impact of CypA or CypB knock-down on replication of Con1. Schematic diagrams of the two Con1-derived constructs are shown in the top. They are composed of the HCV 5' NTR, the IRES of poliovirus for high-level expression of the firefly luciferase gene, the EMCV IRES, the HCV replicase coding region and the 3' NTR. To enhance RNA replication, 3 cell culture adaptive mutations (labelled with asterisks) have been introduced in case of the wild type (ET) replicon [31]. The GND mutant contains an active site mutation destroying NS5B RdRp activity, thus serving as control to measure decay kinetics of luciferase activity expressed from transfected input RNA. Huh7-Lunet cells (left panel) and Huh7.5 cells (right panel) specified below the schematics of the replicons were transfected and replication was analyzed as described in the legend of panel A. One experiment of two independent repetitions, each performed in duplicate is shown.

doi:10.1371/journal.ppat.1000546.g002 
Finally, we studied whether CypA dependence of HCV replication is specific for this virus or applies to other flaviviruses by analyzing replication of a Dengue virus 2 (New Guinea C isolate) replicon in the same cell lines. We found that RNA replication of Dengue virus was not affected by CypA or CypB knock-down in Huh7-Lunet cells (Fig. S1, left panel) whereas in Huh7.5 cells with a CypA knock-down a slight reduction of replication was found (right panel). However, also in this case this difference was at the limit of statistical significance. In summary, we concluded that CypA is an essential host cell factor required for RNA replication of HCV but not Dengue virus, whereas CypB appears to play no role for HCV replication.

To study the impact of these knock-downs on replication of full length HCV genomes we used a bicistronic reporter genome (JclLuc; Fig. 3A, upper panel). It is derived from the highly assembly competent chimera Jcl composed of the genotype 2a isolate J6 and $\mathrm{JFHl}$ that are fused at a distinct site in the N-terminal transmembrane domain of NS2 [32] (Fig. 3A). For this and all subsequent studies phenotypic analyses were performed primarily in Huh7-Lunet cells because of the more potent knock-down of CypA and CypB expression as compared to Huh7.5-derived cell lines (Fig. 1). A very potent inhibition of Jcl-Luc replication was found in CypA, but not CypB knock-down cells (Fig. 3A, lower left panel). In fact, the inhibition was much stronger compared to the one observed with the subgenome and replication did not recover even at later time points. Infectivity was not detected in supernatants of CypA knock-down cells (Fig. 3A, right panel), but not significantly reduced in supernatants of CypB knock-down cells.

The profound inhibition of Jcl-Luc replication in CypA knockdown cells might be due to the less robust replication of this
A
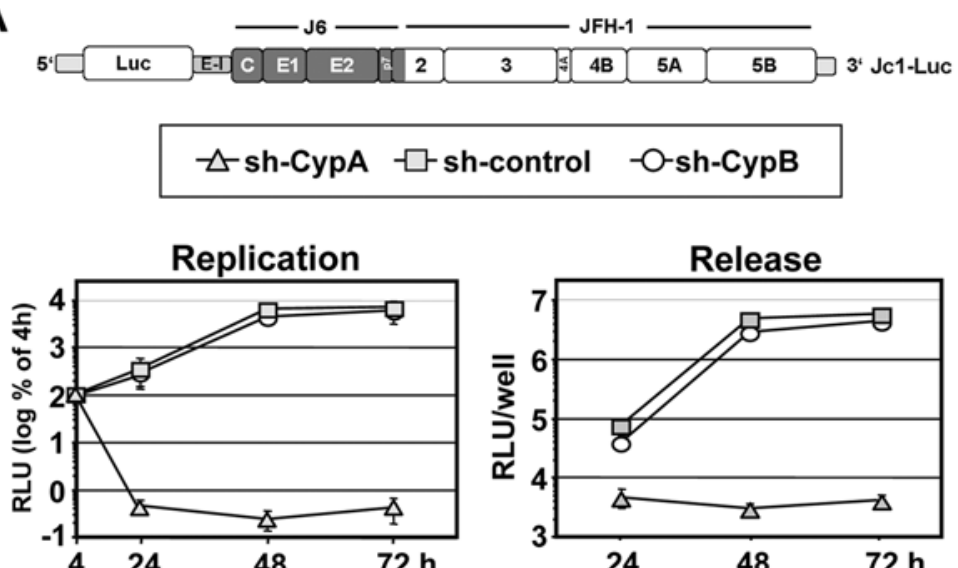

c
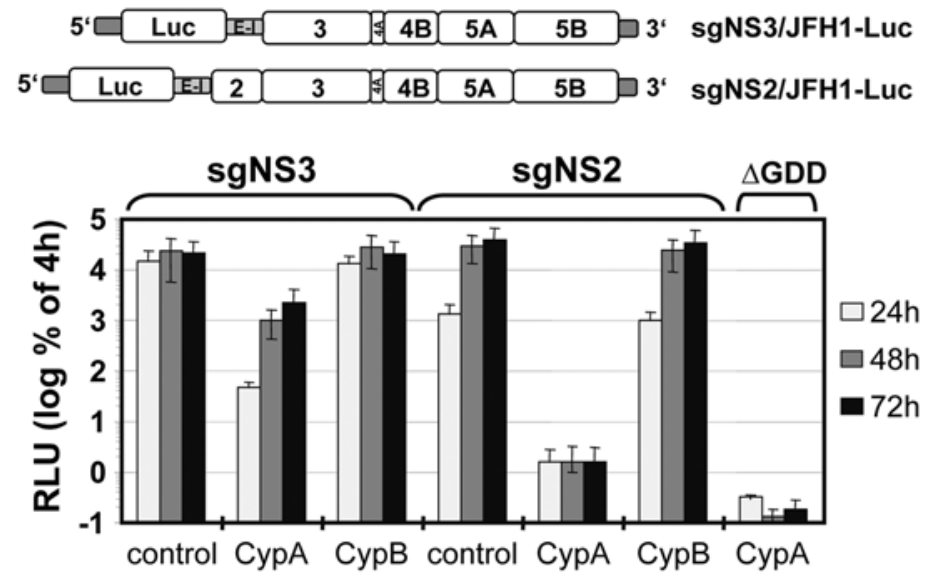

B
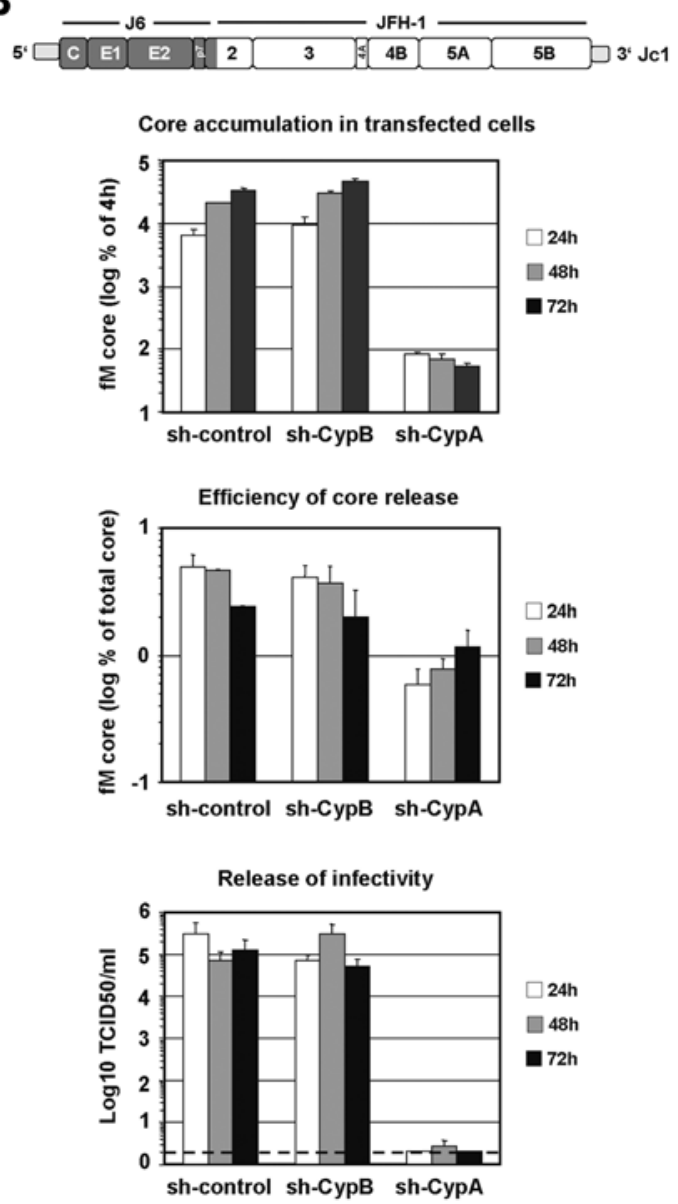

Figure 3. Increased inhibition of RNA replication of JFH1 full length genomes and NS2-containing replicons by CypA knock-down. (A) Huh7-Lunet cells stably transduced with the control vector (sh-control) or the vector encoding the CypA- or CypB-specific shRNA were transfected with the Jc1-Luc genome (shown at the top). Replication of the genome was determined by luciferase assay with cell lysates prepared at given time points (left panel). Infectivity released from transfected cells was measured by inoculation of naive Huh7.5 cells with culture supernatants harvested at given time points after transfection and luciferase activity was determined in infected cells $72 \mathrm{~h}$ after inoculation (right panel). Means and standard deviations of two independent experiments are shown. The dark shaded region in the HCV genome map in the top indicates the J6-derived sequence; the white region corresponds to JFH1. (B) Huh7-Lunet cells were transfected with the Jc1 genome and accumulation of intracellular core protein amounts was determined by core-specific ELISA (upper panel). Kinetic of core release is shown in the middle panel. Core protein in culture supernatants of transfected cells was normalized to total core protein amounts (intra- plus extracellular core). Kinetic of release of infectious virus particles is shown in the bottom panel. Infectivity was determined by $\mathrm{TCID}_{50}$ assay. Background of the assay is indicated by the horizontal dashed line. (C) Huh7-Lunet cells were transfected with JFH1-derived subgenomic replicons encoding an NS3 to 5B or NS2 to 5B replicase of JFH1 (sgNS3/ JFH1-Luc and sgNS2/JFH1-Luc, respectively) and replication was determined by luciferase assay as described in the legend to panel (A). Background of the assay was determined with the sgNS3-derived replicon with an active site mutation destroying NS5B RdRp activity ( $\triangle \mathrm{GDD}$ ). A representative experiment of three independent repetitions, each performed in duplicate is shown.

doi:10.1371/journal.ppat.1000546.g003 
bicistronic genome compared with the sgNS3/JFH1-Luc replicon [33]. Alternatively, CypA dependence of JFH1 full length HCV genomes and subgenomes may differ. We addressed these possibilities by transfection of CypA knock-down cells with a full length genome lacking heterologous sequences. Owing to highest assembly efficiency we used the Jcl chimera (Fig. 3B). Viral replication was scored by determination of intracellular core protein amounts whereas virus production was determined by measurement of core released into the supernatant of transfected cells as well as infectivity titers in culture supernatant (TCID ${ }_{50}$; see materials and methods). Replication of the Jcl genome was potently reduced in CypA knock-down cells, but unaffected by CypB knock-down. In addition to impaired replication, core release was also reduced as deduced from the relative core protein amounts in cell lysate and culture supernatant. In agreement with this profound block of replication and impaired assembly no infectivity was detected in supernatants of CypA knock-down cells even though virus titers in control cells were in the range of $10^{5}$ $\mathrm{TCID}_{50} / \mathrm{ml}$ and higher.

The congruent results obtained with the genomic reporter replicon and the Jcl genome suggest that CypA targets an additional viral factor outside of the minimal replicase (NS3 to NS5B). We assumed that NS2 would be the most likely candidate for three reasons. First, cleavage at the NS2-3 site by NS2 is essential for RNA replication [34]; second, the structural proteins core, E1 and E2 as well as p7 are dispensable for RNA replication [35]; third, NS2 is required for HCV assembly [12,13]. To put this assumption to the test we performed comparative transient replication assays by using subgenomic JFH1 replicons encoding either an NS2 to NS5B or NS3 to NS5B replicase (sgNS2/JFH1Luc and sgNS3/JFH1-Luc, respectively) (Fig. 3C). Replication of the sgNS2 replicon was almost completely blocked in Huh7-Lunet cells with a CypA knock-down and only slightly above background as determined with the replication defective NS5B active site mutant JFH1/ $\triangle$ GDD. In contrast, replication kinetic of the sgNS2 RNA was well comparable between Huh7-Lunet cells with a CypB knock-down and control cells that had been transduced with a non-targeting luciferase shRNA. We note a lower replication of the sgNS2 replicon in CypB knock-down cells at $24 \mathrm{~h}$ post transfection as compared to the sgNS3 replicon, but this reduction was also observed with control cells (Fig. 3G). At later time points, replication of $\operatorname{sgNS} 2$ and $\operatorname{sgNS} 3$ replicons was comparable. In summary these results provide genetic evidence that CypA also targets a factor outside of the replicase, most likely NS2.

\section{Isomerase activity of CypA is essential for HCV RNA replication}

Although the results described so far clearly support an important role of CypA for HCV replication, we could not exclude that the observed phenotype was due, at least in part, to off-target effects. For this reason, we transduced the Huh7-Lunet CypA knock-down cell lines with a CypA expression construct that was not targeted by the shRNA ('rescue' cell line in Fig. 4). In addition, we generated CypA knock-down cell lines expressing a CypA mutant (H126Q) that retains less than $1 \%$ of wild type isomerase activity [36,37]. As shown in Fig. 4A and B, CypA expression levels achieved upon transduction of the shRNAresistant CypA genes was very high (Fig. 4A). In fact, by using quantitative immunofluorescence we found that expression level of CypA in wild type 'rescue' cell lines was about two-fold higher as compared to sh-control cells $(234+/-4.7$ counts per cell based on 8-bit gray value histograms, generated with the ImageJ software package, versus $125+/-25.3$ counts per cell, respectively) see materials and methods). Knock-down of CypA had no effect on expression levels of CypB (Fig. 4B, lane 7) and vice versa (data not shown). Upon transfection of CypA wt 'rescue' cells with sgNS3/ JFH1-Luc, RNA replication was fully restored (Fig. 4G) thus excluding off-target effects of the shRNA that was used for silencing of CypA expression. However, no rescue was obtained in cells stably transduced with the H126Q mutant (Fig. 4C), arguing that isomerase activity of CypA is essential for HCV RNA replication.

\section{CypA is the target for antiviral activity of the cyclosporine analogue DEBIO-025}

To gain further insight into the role of CypA for replication of $\mathrm{HCV}$, we wanted to take advantage of the fact that cyclosporine (CsA) binds to and sequesters CypA [4] and causes a profound inhibition of $\mathrm{HCV}$ replication [25,38]. Moreover, we have recently shown that the cyclosporine analogue DEBIO-025 also inhibits replication of $\mathrm{HCV}$, but the cellular target of this compound was not clear [5]. To clarify this question we first compared the antiviral activity of CsA and DEBIO-025 side-byside in a JFH1 replicon system. Cells transfected with the sgNS3/ JFH1-Luc replicon that expresses the firefly luciferase (Fig. 2A, upper panel) were treated $4 \mathrm{~h}$ post transfection for $72 \mathrm{~h}$ with various concentrations of CsA or DEBIO-025. Cells were then lysed and luciferase activity was determined. The JFH1 subgenome was potently inhibited both by CsA and DEBIO-025, with the latter having a higher antiviral activity (about 3-4 fold lower $\mathrm{IC}_{50}$ and $\mathrm{IC}_{90}$ (Fig. 5A)).

We next determined whether CypA is targeted by DEBIO-025 using transient replication assays with the same subgenomic JFH1 RNA. Transfected cells were treated with various concentrations of DEBIO-025 and after $72 \mathrm{~h}$ luciferase activity contained in cell lysates was determined. The results depicted in Fig. 5B show that HCV replication was much more sensitive to DEBIO-025 treatment in CypA knock-down cells whereas $\mathrm{IC}_{50}$ and $\mathrm{IC}_{90}$ values determined in CypB knock-down cells were comparable to naïve and control shRNA-transduced cells. Moreover, when doseresponse assays were performed with CypA wild type 'rescue' cell lines, DEBIO-025 sensitivity was reduced even to beyond the level determined for naive cells (Fig. 5C). This enhanced resistance is probably due to the elevated expression levels of CypA in the rescue cell line as compared to naïve cells. Most importantly, DEBIO-025 sensitivity was not affected by transduction of the gene containing the CypA isomerase mutation (H126Q) suggesting that an enzymatically active CypA is required for HCV RNA replication or that this CypA protein binds DEBIO-025 with lower efficiency. In summary, our results show that CypA is the target for the antiviral activity of DEBIO-025 and that isomerase activity of CypA appears to be required for HCV replication.

\section{A mutation in the C-terminus of NS5A confers DEBIO-025 resistance and reduces dependence of HCV RNA replication on CypA}

Given the finding that DEBIO-025 can be used as a convenient tool for the pharmacological inhibition of CypA and to study its role in HCV RNA replication, we sought to identify the target of CypA in the HCV replicase by selection for DEBIO-025 resistance. Due to the high potency of DEBIO-025 and the small window between $\mathrm{IC}_{50}$ and $\mathrm{IC}_{90}(0.2 \mu \mathrm{g} / \mathrm{ml}$ and $0.5 \mu \mathrm{g} / \mathrm{ml}$; Fig. $5 \mathrm{~A})$, attempts to select for resistance by using virus passage or cells persistently infected with Jcl were not successful. Concentrations in the range of the $\mathrm{IC}_{90}$ or above lead to rapid elimination of $\mathrm{HCV}$ from cell cultures and treatment with a dose corresponding to the $\mathrm{IC}_{50}$ did not sufficiently affect replication. We therefore utilized a selectable 
A

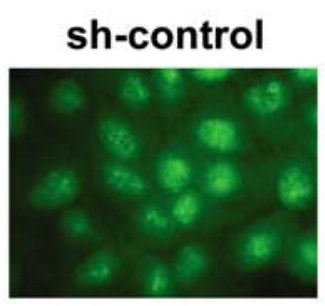

\section{sh-CypA wt- rescue}
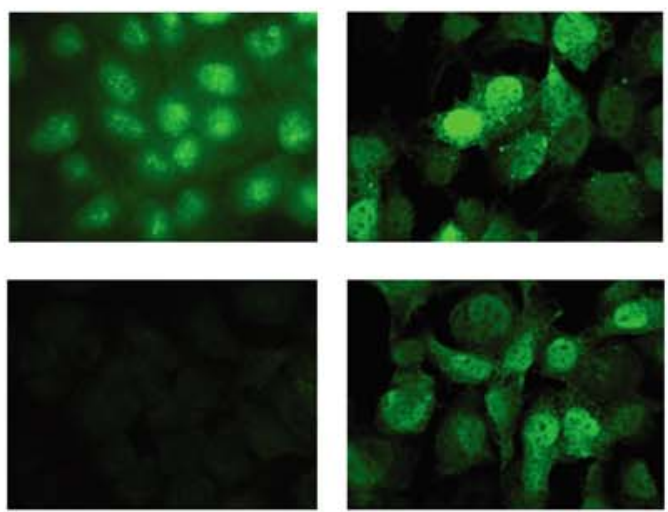

sh-CурA

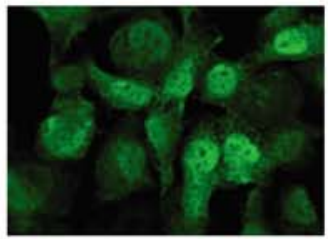

sh-СурA H126Qrescue

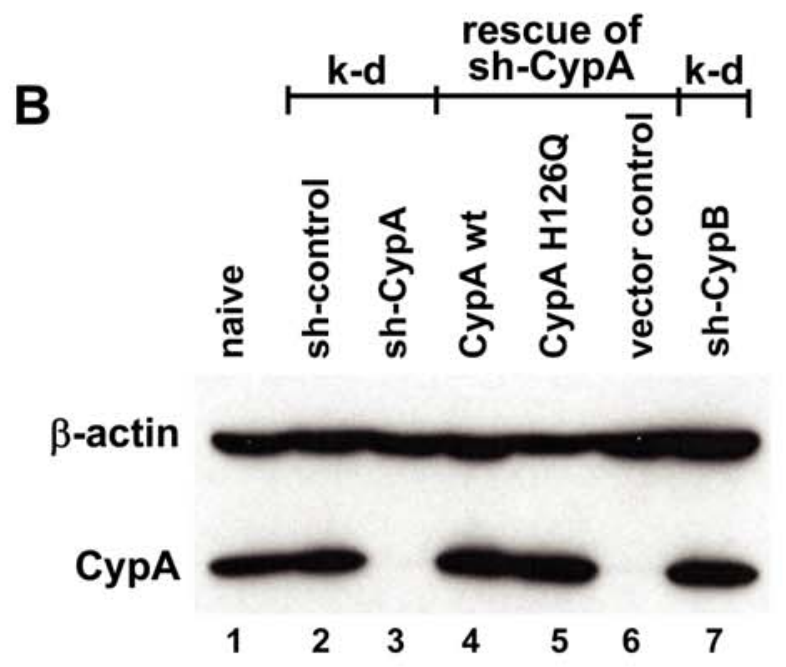

C

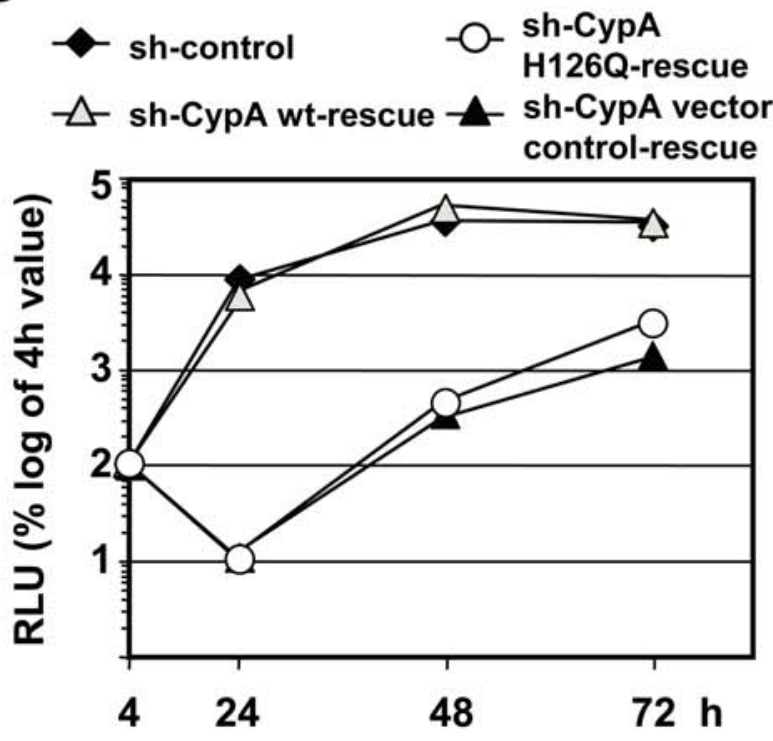

Figure 4. Rescue of HCV replication by overexpression of CypA wt but not CypA H126Q in stable knock-down cells. (A) Huh7Lunet cells with a stable knock-down of CypA (lower left) were stably transduced with a CypA wild type (wt) expression construct (CypA wtrescue; upper right) or a CypA expression construct carrying the $\mathrm{H} 126 \mathrm{Q}$ mutation (CypA H126Q-rescue; lower right) that are both resistant to the shRNA. For comparison, Huh7-Lunet cells stably transduced with the same expression vector containing the non-related control shRNA (sh-control) are shown (upper left). Cells were analyzed by immunofluorescence using a CypA-specific antibody. Note that CypA expression levels are higher in the 'rescue cells' as compared to the vector control cells (sh-control). (B) Lysates from cells specified in the top were analyzed by Western blot using a CypA-specific antibody. Lane 6 shows a Western blot of a lysate of Huh7-Lunet cells with a stable knock-down of CypA and transduced with an empty vector (cell line sh-CypA vector control-rescue). Beta-actin was used as a loading control. K-d, knockdown; rescue, stable CypA knock-down cells transduced with CypA wt or the $\mathrm{H} 126 \mathrm{Q}$ mutant or empty expression vector, respectively. Loaded samples are specified above each lane. (C) Restoration of HCV replication by ectopic expression of CypA wt, but not CypA H126Q in stable CypA knock-down cells. Huh7-Lunet cells specified in the top were transfected with a bicistronic JFH1 reporter replicon (sgNS3/JFH1Luc) and RNA replication was determined by luciferase assay 24,48 and $72 \mathrm{~h}$ post transfection. Values are normalized for transfection efficiency by using the $4 \mathrm{~h}$ value. Means and standard deviations of a representative experiment of two independent repetitions are shown. doi:10.1371/journal.ppat.1000546.g004

JFH1-derived subgenome that carried a stable in-frame insertion of the red fluorescent protein (RFP) in domain 3 of NS5A [39], which allowed visualization of replicating $\mathrm{HCV}$ via RFP autofluorescence in live cells. Huh7-Lunet cells were transfected with this sgNS3/ JFH1-neo/RFP replicon and cultured in double-selection medium containing $500 \mu \mathrm{g} / \mathrm{ml}$ of G418 and 0 or 0.5 or $1 \mu \mathrm{g} / \mathrm{ml}$ of DEBIO025. After 18 cell passages, we had selected for a DEBIO-025 resistant cell pool with a calculated $\mathrm{IC}_{50}$ of about $1 \mu \mathrm{g} / \mathrm{ml}$ corresponding to an about 5 -fold lower sensitivity as compared to non-selected cells (see Fig. 5A).

To identify mutations that confer increased DEBIO-025 resistance and thus presumably reduced CypA dependence, replicons present in two independent RNA preparations of the cell pool were prepared from DEBIO-025 selected cells. From each RNA preparation the complete $\mathrm{HCV}$ coding region was amplified in two overlapping fragments (Fig. 6A) and 3 or 4 molecular clones of each fragment were subjected to nucleotide sequence analysis, respectively. As summarized in Table 1 and Fig. 6A, 4 mutations were identified residing in the NS3 helicase domain $(\mathrm{Y} 1421 \mathrm{~F})$ or at various positions in NS5A. Two of these were mapped to domain 2 of NS5A (D2229G, L2266F) and one to the very C-terminus of domain 3 (V2440A). Quite surprisingly, we have recently identified position 2440 , which is close to the NS5AB cleavage site, as a major determinant of HCV assembly [40]. However, in case of this virus titer enhancing mutation (TEM) the substituting amino acid residue was leucine instead of the alanine found in the DEBIO-025 resistance selection.

Multiple sequence alignments covering $\mathrm{HCV}$ isolates of all genotypes as deposited in the European HCV database (euHCVdb) [41] revealed that all positions identified in DEBIO025 selected replicons are polymorphic (Table 1). The mutations residing in the NS3 helicase and at the C-terminus of domain 3 of NS5A appear to be natural variants, because the substituting residues (phenylalanine in case of the helicase and alanine in case of NS5A) are present in patient isolates (Table 1). Other substituting residues are naturally found in case of the two mutations in domain 2 of NS5A.

The impact of these 4 selected mutations on DEBIO-025 sensitivity was determined by transferring each mutation individ- 
A

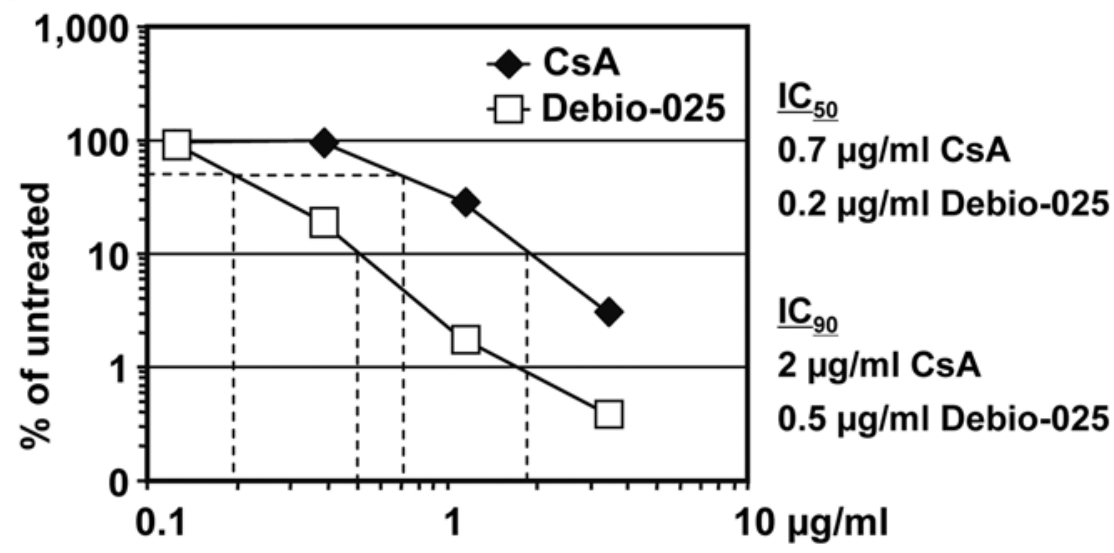

B

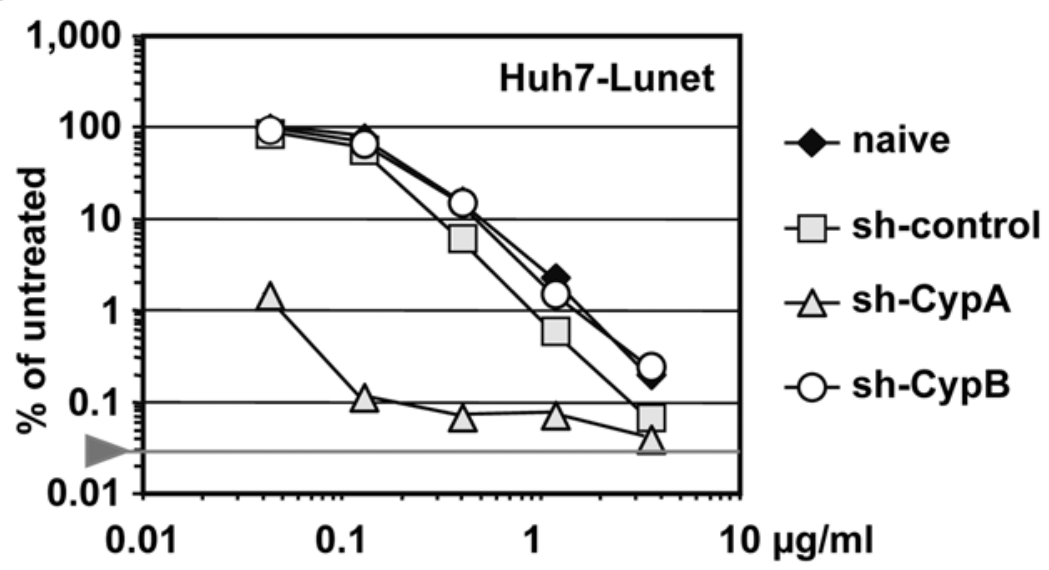

C

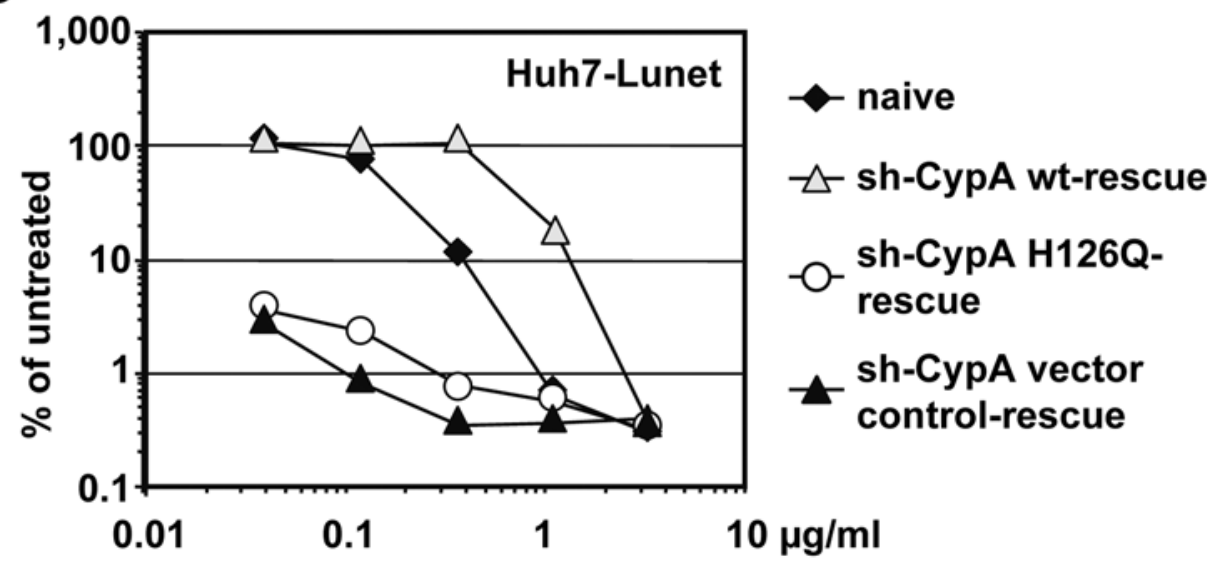

Figure 5. CypA is the target of the cyclosporine analogue DEBIO-025. (A) Dose-response curve for cyclosporine A (CsA) and DEBIO-025. Huh7-Lunet cells transfected with JFH1 reporter replicon (sgNS3/JFH1-Luc, shown in the top of Fig. 2A) were incubated $4 \mathrm{~h}$ post electroporation with escalating concentrations of CsA or DEBIO-025 and $72 \mathrm{~h}$ later cells were lysed and luciferase activity was determined. (B) Naive Huh7-Lunet cells or cells transduced with a retroviral vector encoding a CypA- or CypB-specific shRNA or transduced with the retroviral vector control (sh-control) were transfected with a HCV luciferase reporter replicon (sgNS3/JFH1-Luc). Four hours post transfection various concentrations of CsA or DEBIO-025 were added, cells were incubated for $72 \mathrm{~h}$ and lysed. Luciferase activities were determined and normalized to the values obtained with mock-treated cells, which was set to $100 \%$. The background of the assay is indicated with the grey horizontal line. (C) Huh7-Lunet cells with a stable knock-down of CypA and transduced with the empty vector (control-rescue) or the shRNA-resistant CypA wt or CypA H126Q encoding vector were transfected with sgNS3/JFH1-Luc. Thereafter cells were treated with various concentrations of DEBIO-025 and $72 \mathrm{~h}$ later, cells were lysed and luciferase activity was determined. Naive Huh7-Lunet cells were analyzed in parallel. (A-C) Means and standard deviations of a representative experiment (2 independent repetitions) are shown.

doi:10.1371/journal.ppat.1000546.g005 
A

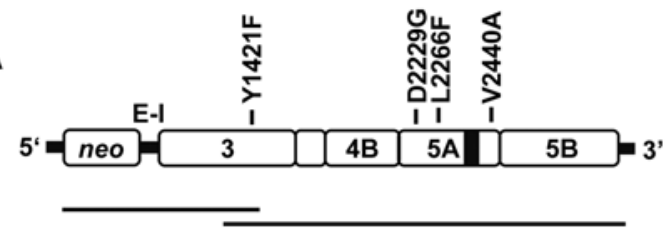

B

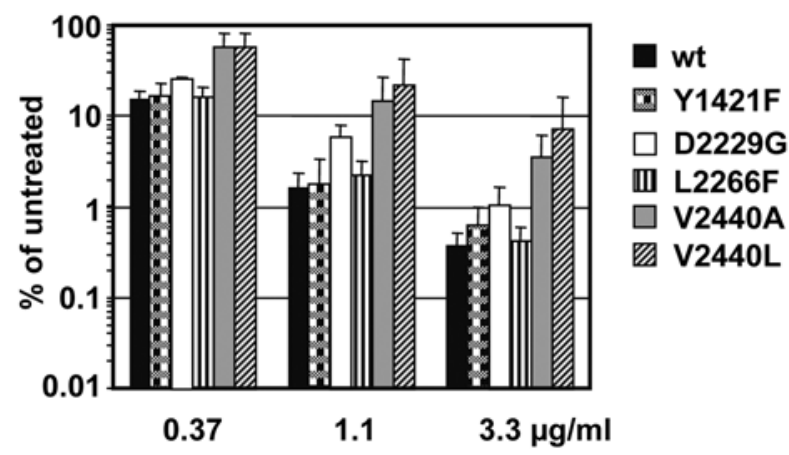

C
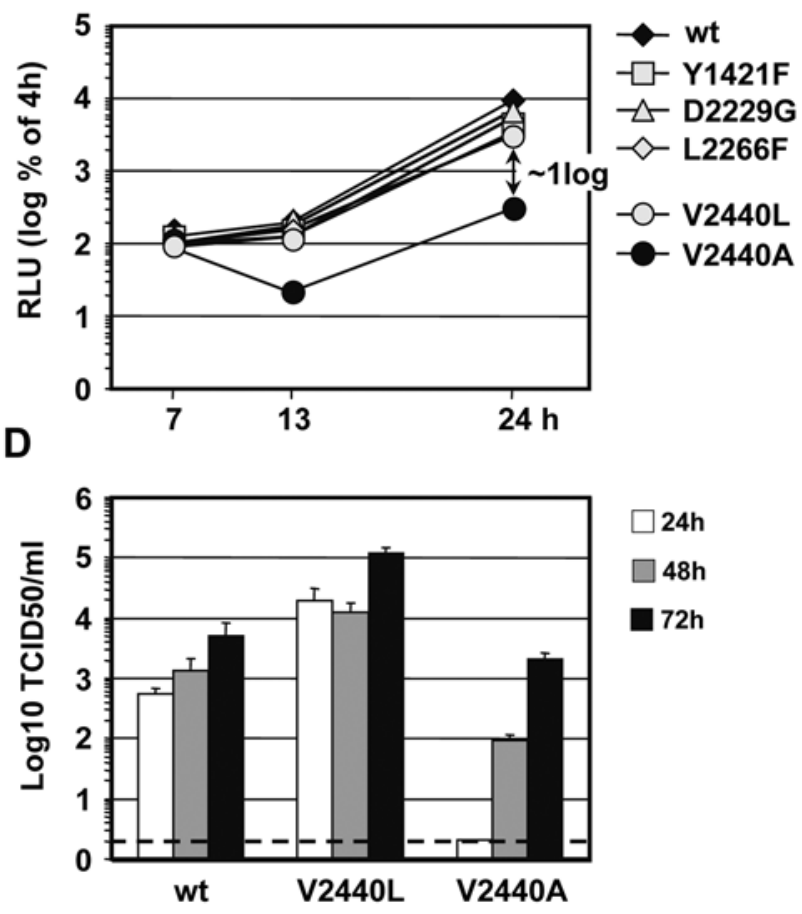

Figure 6. Identification and characterization of mutations conferring resistance against the antiviral activity of DEBIO025. (A) Design of the selectable JFH1 replicon that was used for the selection of DEBIO-025 resistance. The construct contains the gene encoding the neomycin phosphotransferase (neo) under control of the HCV IRES whereas the replicase gene encoding the NS3 to NS5B polyprotein fragment is translated under control of the EMCV IRES (E-I). The sequence encoding the red fluorescent protein (RFP; shown as black bar in NS5A) was inserted in frame into domain 3 of NS5A to facilitate detection of HCV during the selection procedure. Mutations identified in replicons that were isolated after 18 passages of cells in double selection medium (G418 and DEBIO-025) are given above the respective position in the polyprotein. Numbers refer to the polyprotein sequence of JFH1. Lines in the bottom refer to the amplicons used for nucleotide sequence analyses. Note that mutations depicted in the top all resided in the 3' amplicon. (B) Dose-response assay of all mutations identified in double selected replicons using the transient sgNS3/JFH1Luc replicon assay. Included is the V2440L mutation recently shown to enhance virus titers without affecting RNA replication [40]. Means and standard deviations of three independent experiments are shown. (C) Replication competence (fitness) of sgNS3/JFH1-Luc replicons (shown at the top of Fig. 2A) carrying one of the mutations specified in the right in comparison to the wild type replicon (wt). Huh7-Lunet cells were transfected with a given replicon and RNA replication was determined by luciferase assay at given time points after transfection. Owing to the limitation of JFH1 replication by the host cell at later time points post transfection, only the early time points are shown. (D) Release of infectivity from Huh7-Lunet cells transfected with the JFH1 wild type genome (wt) or JFH1 containing the V2440L or the V2440A mutation. Supernatants from transfected cells were harvested at given time points and infectivity titers were determined by $\mathrm{TCID}_{50}$ assay. The background of the assay is indicated with the horizontal dashed line. (C, D) Means and standard deviations of a representative experiment (3 or 2 independent repetitions, respectively) are shown.

doi:10.1371/journal.ppat.1000546.g006

ually into a wild type JFH1 luciferase replicon (sgNS3/JFH1-Luc; Fig. 2A) and determining replication fitness (Fig. 6C) as well as DEBIO-025 sensitivity (Fig. 6B). In this and all subsequent analyses we included the TEM V2440L, because it resides at the very same position like the putative resistance mutation V2440A. The results in Fig. 6B demonstrate that only the mutations affecting residue 2440 in NS5A reduced DEBIO-025 sensitivity to a significant extent. In fact, the $\mathrm{IC}_{50}$ of replicons containing the V2440A or the V2440L substitution was increased about 5- to 10fold (Fig. 6B) whereas the mutations in domain 2 of NS5A had either minor or no effect. Replication fitness of only the V2440A mutant was reduced (about 10-15-fold as compared to the wild type) whereas all other mutants were unaffected (Fig. 6C).

Since V2440L is a TEM, we next assessed the impact of this and the V2440A substitution on virus production. The two mutations were introduced into the $\mathrm{JFHl}$ wild type genome and virus titers released from cells at various time points after transfection were determined (Fig. 6D). In agreement with our earlier report [40] the V2440L substitution enhanced virus production without affecting RNA replication, whereas the V2440A substitution reduced virus production to an extent corresponding to the lower replication. These results suggest that only V2440L is a TEM.

The data described so far show that CypA is an important cellular determinant of HCV RNA replication and they suggest that DEBIO-025 blocks HCV replication by interfering with CypA. Having identified V2440L/A as DEBIO-025 resistance conferring mutations we assumed that they might render $\mathrm{HCV}$ replicons less dependent on CypA. To corroborate this assumption we performed transient replication assays with JFHl wild type, JFH1 V2440L and JFH1 V2440A sgNS3-replicons in CypA knock down cells (Fig. 7A and $\mathrm{B}$, respectively). In line with our assumption we found that replication of the V2440L mutant was less impaired in CypA depleted cells. The analogous result was found when we scored replication by immunofluorescence of transfected cells (not shown). In case of the V2440A mutation, due to reduced replication fitness only a slight but statistically significant difference $(p<0.002, \alpha=0.05$; for values $72 \mathrm{~h}$ and $96 \mathrm{~h}$ post transfection, respectively) compared to the wt replicon was apparent (Fig. 7B). These data suggest that the mutations close to the C-terminus of NS5A conferring DEBIO-025 resistance render HCV replication less dependent on CypA.

Correlation between cleavage kinetic at the NS5A-B site and CypA dependence

We have recently shown that the V2440L substitution leads to delayed cleavage at the NS5A-B site [40]. Having identified another substitution at the same site that also confers DEBIO-025 resistance and lower dependence on CypA (V2440A), we wondered whether 
Table 1. Mutations identified in JFH1/RFP replicons after selection for DEBIO-025 resistance.

\begin{tabular}{llll}
\hline & & & \\
\hline HCV protein & Substitution frequency & Amino acid substitution & Amino acid appearance \\
\hline NS3 helicase & $7 / 7$ & Y $1421 \mathrm{~F}(391)$ & Y, F \\
NS5A domain II & $5 / 7$ & D $2229 \mathrm{G}(253)$ & $\mathrm{D}, \mathrm{E}, \mathrm{A}$ \\
NS5A domain II & $7 / 7$ & $\mathrm{~L} 2266 \mathrm{~F}(290)$ & $\mathrm{E}, \mathrm{L}, \mathrm{R}, \mathrm{H}, \mathrm{M}, \mathrm{D}, \mathrm{N}, \mathrm{G}$ \\
NS5A domain III & $7 / 7$ & V $2440 \mathrm{~A}(464)$ & V, A, I \\
NS5A domain III & n.a. & V 2440 L (464) & V, A, I \\
\hline
\end{tabular}

aNumber of DNA clones in which the mutation was found vs. number of clones that were analyzed. Note that all mutations were found with the $3^{\prime}$ PCR fragment (Fig. 6A) and therefore the identified mutations coexisted in the same replicon RNA.

${ }^{b}$ Amino acids found at the corresponding position of the polyprotein of HCV isolates available in the European HCV database and belonging to genotypes $1 \mathrm{a}, \mathrm{b}, \mathrm{c}$; 2a,b,c,i,k; 3a,b,k; 4a,d,f; 5a; 6a,b,c,e,f,g,h,i,j, k,l,m,n,p; 7a.

'Virus titer enhancing mutation; data taken from [40].

n.a., not applicable.

Bracketed numbers refer to positions of the individual HCV protein of the JFH1 isolate.

doi:10.1371/journal.ppat.1000546.t001

this mutation affects cleavage kinetics as well. We therefore performed pulse-chase experiments using a T7-based expression system. Huh7-Lunet cells stably expressing the T7 RNA polymerase were transfected with NS3 to NS5B polyprotein expression constructs corresponding to the wild type or containing the V2440L or the V2440A mutation. Proteins were radiolabeled metabolically with $\left[{ }^{35} \mathrm{~S}\right]$ methionine/cysteine for 90 minutes and treated with nonradioactive medium for one or two hours. Cells were lysed and NS5A- and NS5B-containing proteins were isolated by immunoprecipitation with mono-specific antibodies (Fig. 8A and B, respectively; a quantification of the autoradiogram shown in panel $\mathrm{A}$ is given in panel $\mathrm{C}$ ). We found that both mutations slowed down cleavage kinetics at the NS5A-B site, with the V2440A mutation causing a much stronger delay of cleavage than the leucine substitution (Fig. 8A, $\mathrm{B}$, lanes 5-7). Best visible was the accumulation of an uncleaved NS5AB precursor protein as well as a precursor with an apparent molecular weight of about $175 \mathrm{kDa}$ corresponding most likely to uncleaved NS4B5AB [17]. Only small amounts of these precursors were found in case of the wild type reflecting faster cleavage at the NS5A-B site. These results suggest a link between processing kinetic at this site and CypA dependence of HCV replication.

To substantiate this observation, we generated additional mutants affecting the NS5A-B cleavage site. Selection of these mutations was guided by a multiple alignment of amino acid residues at the P7-P1 positions of the NS5A-B cleavage site in HCV isolates deposited in the European HCV database (euHCVdb) [41] (Fig. 9A). Interestingly, amino acids at positions $\mathrm{P} 7-\mathrm{P} 4$ of the $\mathrm{JFH} 1$ isolate are rather unique amongst all the other genotypes including genotype 2 (Fig. 9A, upper half). In case of genotype 2a the P5 and P4 residues are serine and valine, respectively. Genotype $2 \mathrm{~b}$ isolates have in addition a P7 glutamic acid residue and a P3 isoleucine residue. Based on this alignment we constructed two cleavage site mutants corresponding to the NS5A-B site of genotype 2a and (with the exception of the $\mathrm{P} 3$ isoleucine) to genotype $2 \mathrm{~b}$ (Fig. 9A, lower half). In addition these mutations were combined with the P3 leucine substitution (V2440L) to determine its contribution on DEBIO-025 resistance, CypA dependence and cleavage kinetics in the context of the $2 \mathrm{a}$ and $2 \mathrm{~b}$ cleavage site. As shown in Fig. 8, cleavage between NS5A and NS5B was enhanced with mutants $2 \mathrm{a}-5 \mathrm{AB}$ and $2 \mathrm{~b}-5 \mathrm{AB}$. A comparison of the protein pattern obtained after immunoprecipitation with the NS5A-specific antibody revealed that the NS5AB precursor was well detectable right after labeling in case of the wild type (Fig. 8A, lane 2) and this protein remained detectable throughout the chase period, albeit at low amounts (lane 3,4). In case of the $2 \mathrm{a}$ and the $2 \mathrm{~b}$ mutants the amounts of this precursor were lower after the $1 \mathrm{~h}$ labeling period and it was not detectable at later time points (lane 11-16). However, upon insertion of the V2440L substitution into each of these mutants, processing was again delayed compared to the wild type and cleavage kinetic was similar to the one observed with the V2440L single mutant (compare lanes 17-22 with 8-10 in Fig. 8A-B and quantification in Fig. 8C).

Upon transfection of Huh7-Lunet cells with replicons containing these mutations, we found that save for the $2 \mathrm{~b}-5 \mathrm{AB}$ mutant, which was slightly impaired, replication of all the other mutants was well comparable to wild type (Fig. 9B). To our great surprise mutants $2 \mathrm{a}-5 \mathrm{AB}$ and $2 \mathrm{~b}-5 \mathrm{AB}$ showed an about 10 -fold increased DEBIO-025 sensitivity (Fig. 9C) and replicated less efficiently in stable CypA knock-down cells (Fig. 9D). However, the analogous mutants containing in addition the V2440L substitution were again less sensitive to DEBIO-025 (Fig. 9C) and replicated more efficiently in CypA knock-down cells (Fig. 9D). These results reveal a correlation between cleavage kinetics at the NS5A-B site, DEBIO-025 resistance and CypA dependence (Table 2). Delay of cleavage kinetics correlates with low DEBIO-025 sensitivity and reduced CypA dependence whereas 'hyper-processing' correlates with increased DEBIO-025 sensitivity and CypA dependence.

Given the link between the V2440L mutation and enhanced $\mathrm{HCV}$ assembly, we tested all cleavage site mutations for their effect on virus production. As shown in Fig. 9E all cleavage site mutations increased virus titers and kinetics. With the exception of the $2 \mathrm{~b}-5 \mathrm{AB}$ V2440L mutant, peak titers were reached already $24 \mathrm{~h}$ post transfection with all mutants and titers were elevated 100- to 1.000 -fold. Especially for the 2a-5AB mutant, already $7 \mathrm{~h}$ after transfection infectious virus was detected in the culture supernatant (compare $2 \mathrm{a}-5 \mathrm{AB}$ also with the very efficient $\mathrm{Jcl}$ in Fig. 9E). A significant, but less pronounced enhancement of kinetics and overall production of virus was achieved with mutant 2b-5AB. When the V2440L substitution was introduced into these mutants, kinetics were reduced, best visible with the $7 \mathrm{~h}$ values (Fig. 9E). In summary, our results reveal a correlation between cleavage kinetics at the NS5A-B site, DEBIO-025 resistance and CypA dependence. The data also suggest that cleavage kinetics at the NS5A-B site and virus assembly may be linked.

\section{Discussion}

Exploitation of host cell machineries to achieve efficient replication is a strategy shared by all viruses. In case of HCV, Cyps have gained increasing interest because of the possibility to interfere pharmacologically with their activity thus providing a new approach for 

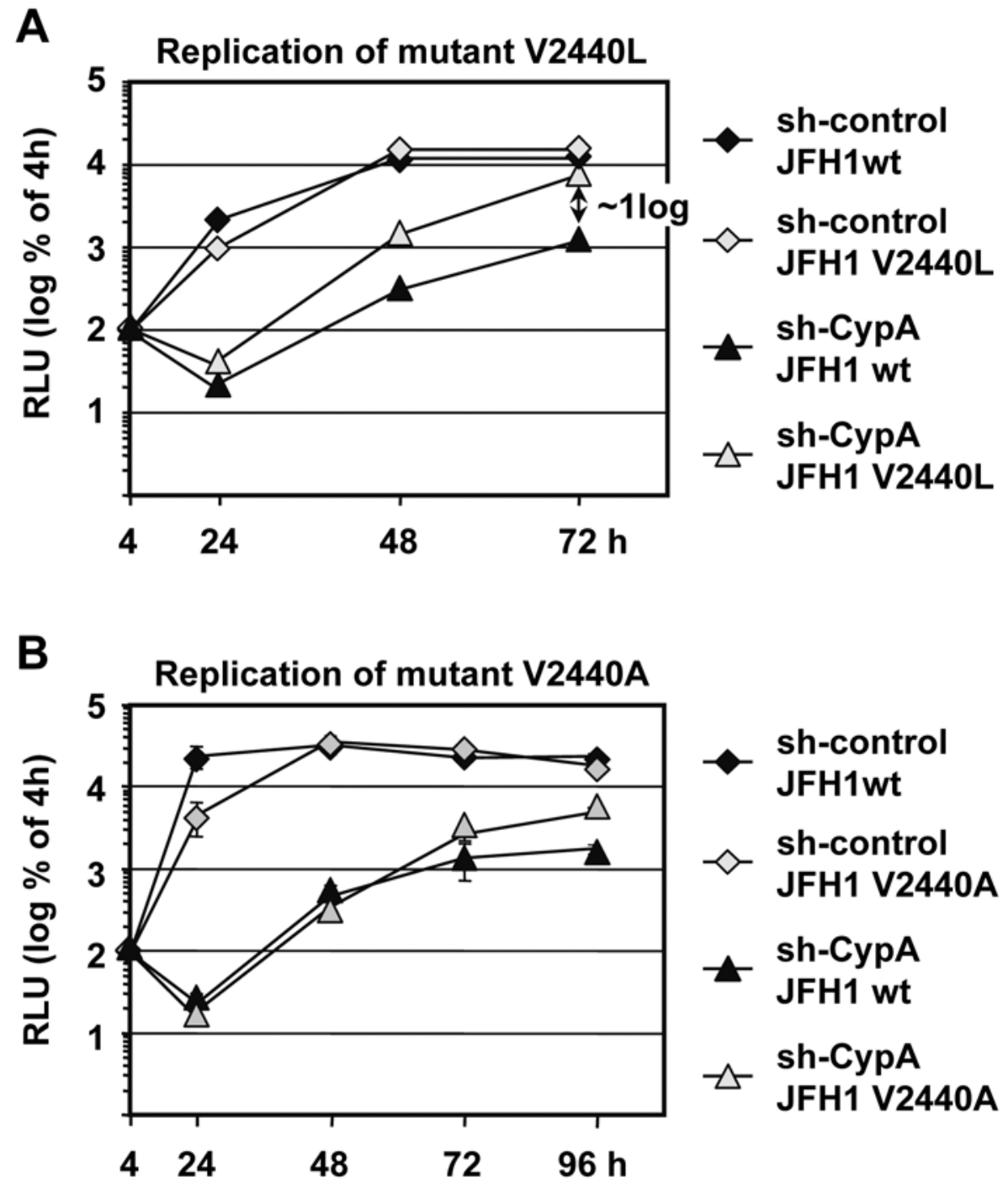

Figure 7. A mutation close to the NS5A/B cleavage site confers resistance to DEBIO-025 and renders HCV replication less dependent on CypA. (A) Replication of sgNS3/JFH1-Luc wt and the V2440L replicon in Huh7-Lunet cells with a stable knock-down of CypA or transduced with the retroviral vector control (sh-control). Cells were lysed at given time points after transfection with each of the replicon RNAs and luciferase activity in cell lysates was determined. Values were normalized to the $4 \mathrm{~h}$ value to exclude differences due to transfection efficiency. Note the higher replication of the V2440L mutant as compared with the wild type in CypA knock-down cells. (B) Experimental setup as in A, with the difference that the V2440A- and not the V2440L-mutant was used. Means and standard deviations of two independent experiments are shown in each panel.

doi:10.1371/journal.ppat.1000546.g007

antiviral therapy [42]. In the present study we demonstrate that CypA but not CypB is the major player for HCV replication. This conclusion is supported by the profound impairment of replication by stable CypA, but not CypB knock-down and the rescue of viral replication by expression of CypA in these cells. Rescue was not achieved by expression of the active site mutant H126Q. This mutation resides in the hydrophobic pocket of CypA and causes a reduction of PPIase activity to about $1 \%$ of the wild type [36,37]. Although this result argues for a role of PPIase activity in HCV replication, it should be kept in mind that CypA mutants that are impaired in isomerase activity have reduced substrate binding $[37,43]$. Thus, PPIase activity and substrate binding cannot be separated genetically and therefore, the exact role of isomerase activity in $\mathrm{HCV}$ replication remains to be determined.

Cyps are a diverse group of proteins that share a PPIase activity and a 109 amino acids long Cyp-like domain [23]. There are 7 major Cyps in humans that differ in their N- and C-terminal sequences, which determine the intracellular localization of a given Cyp. For instance, CypA (as well as Cyp40 and CypNK) are cytosolic whereas $\mathrm{CypB}$ and $\mathrm{CypC}$ possess N-terminal signal sequences, which target the proteins to the ER-resident secretory machinery. CypD has a signal sequence recruiting the protein to mitochondria and CypE is localized in the nucleus. Although the role of Cyps in HCV replication has been established in several reports, conflicting results exist as to which individual Cyp is required. Two studies identified CypB, but not CypA, as the key player in HCV replication [24,25]. One study describes that CypA, B and C are required [44] and the study by Yang and coworkers suggests that CypA, but not $\mathrm{CypB}$ and $\mathrm{C}$ is critical for HCV replication [26]. The results presented in this report are in full support of the work by Yang and colleagues. We also identify CypA as the key player in HCV replication and show that this host 
A

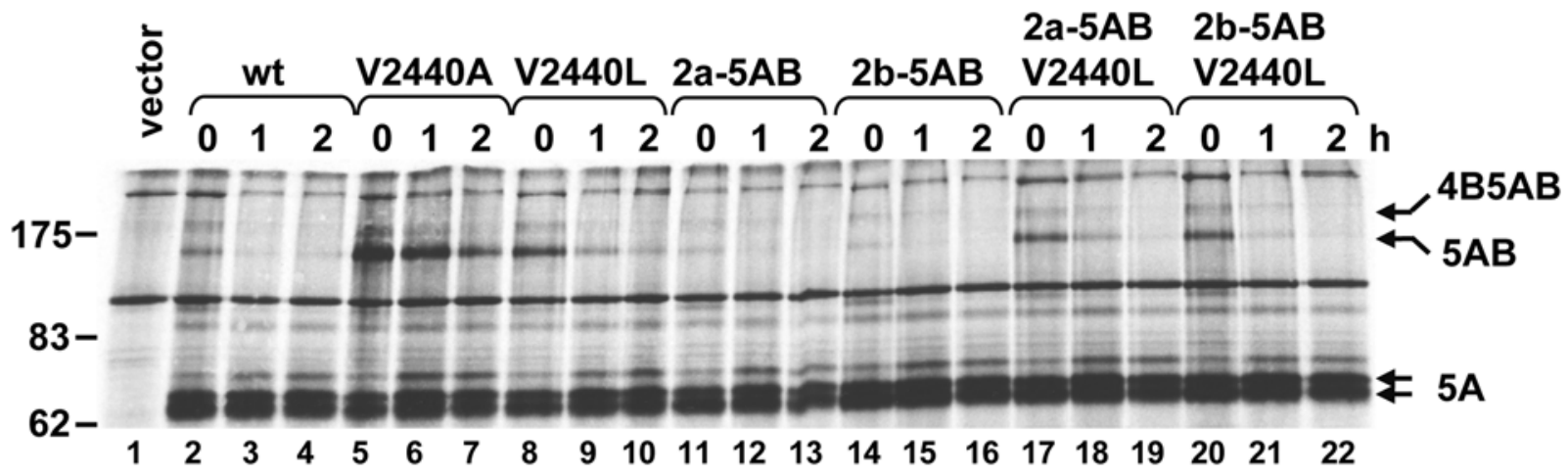

B
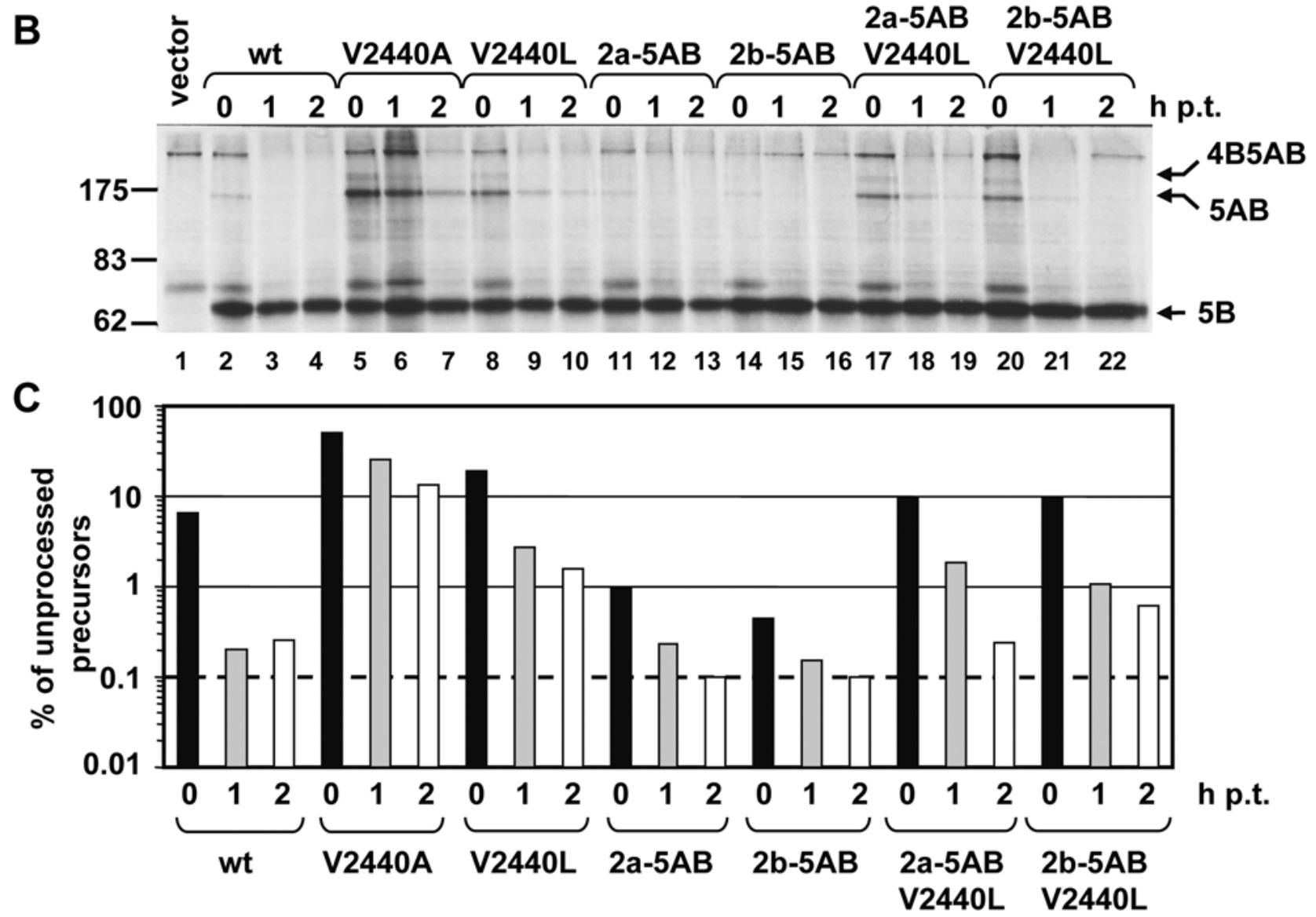

Figure 8. Alteration of the kinetics of polyprotein processing at the NS5A-B cleavage site by mutations conferring DEBIO-025 resistance. (A, B) Huh7-Lunet/T7 cells were transfected with subgenomic NS3 to NS5B JFH1 expression constructs derived from the wild type or mutants specified at the top of each panel or with the empty vector (pTM1-2; lane 1). After $24 \mathrm{~h}$ cells were pulse-labeled for $90 \mathrm{~min}$ with $\left[{ }^{35} \mathrm{~S}\right]$ methionine/cysteine. Cells were lysed immediately (0) or incubated with non-radioactive medium for 1 or 2 additional hours. Immunoprecipitation was performed using either an NS5A- (A) or NS5B-specific antibody (B). Molecular weights are given on the left, arrows to the right point to the respective HCV proteins detected. (C) Amounts of unprocessed NS5AB and NS4B5AB precursors were quantified by phosphoimaging. Values obtained with the phosphoimage displayed in the upper panel are shown in panel C. Similar ratios were obtained with the phosphoimage of the NS5B-specific immunoprecipitation (data not shown).

doi:10.1371/journal.ppat.1000546.g008

factor is the target of the cyclosporine analogue DEBIO-025. The reasons for the discrepant results is not known, but may in part be due to the use of different cell systems, HCV isolates or experimental conditions such as utilized siRNAs. However, we note that in our study HCV replication was impaired upon CypA knock-down both in Huh7-Lunet and Huh7.5 cells; two widely used Huh7-derived cell clones that are highly permissive for HCV replication. We also note that the impairment of HCV replication was found both with the JFH1 and the Conl isolate similar to what Yang and colleagues described [26]. In support of these findings, a very recent study also describes CypA as the main actor in HCV replication [45]. Considering the subcellular localization, the cytosolic CypA is more likely to interact with the HCV replicase that also resides on the cytosolic side of the ER membrane, in contrast to the ER luminal CypB. In addition, CypB and $\mathrm{C}$ are expressed 10-150-fold lower in hepatoma cells as 
A

\begin{tabular}{|c|c|c|}
\hline \\
\hline & con & --sv--- \\
\hline & con & E-SVI- \\
\hline & H77 & TEDV--- \\
\hline & Con1 & SEDV--- \\
\hline & con & EQSV-- \\
\hline & $\mathrm{b}$ con & -EDV- \\
\hline \multicolumn{3}{|c|}{ CONSTRUCTS } \\
\hline & & DTTVC \\
\hline $440 \mathrm{~L}$ & & $--L-$ \\
\hline$-5 A B$ & & $--s v-$ \\
\hline$-5 A B$ & & -sv-. \\
\hline$-5 A B$ & V2440I & -SVL \\
\hline$-5 A B$ & V2 $440 \mathrm{~L}$ & $-s y$ \\
\hline
\end{tabular}

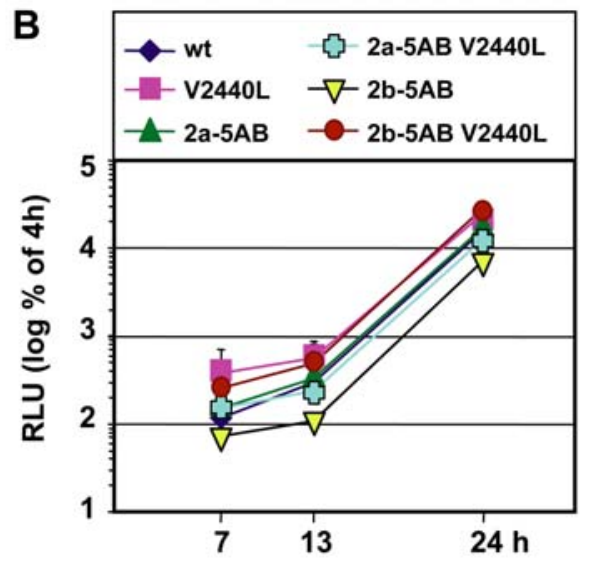

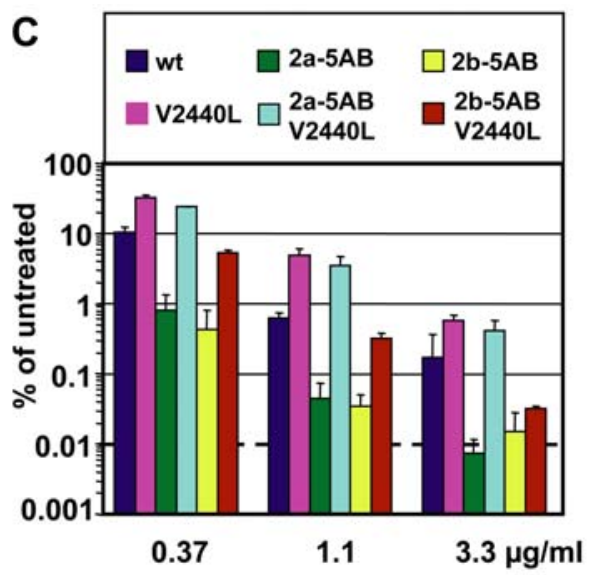

D

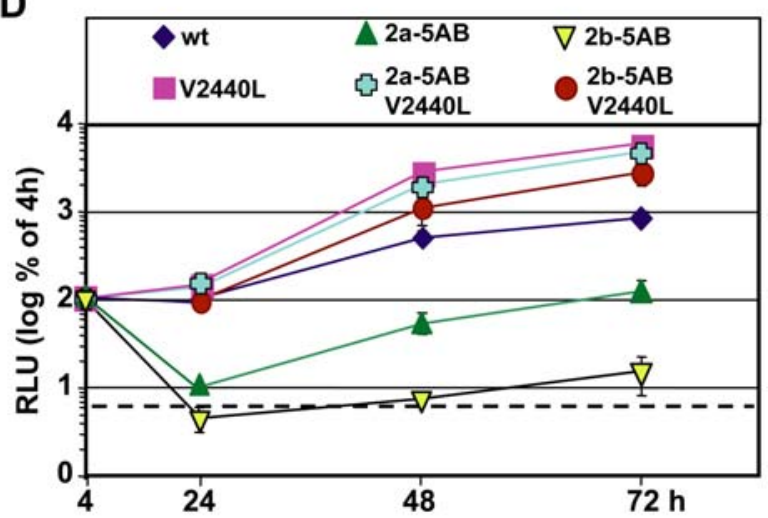

E

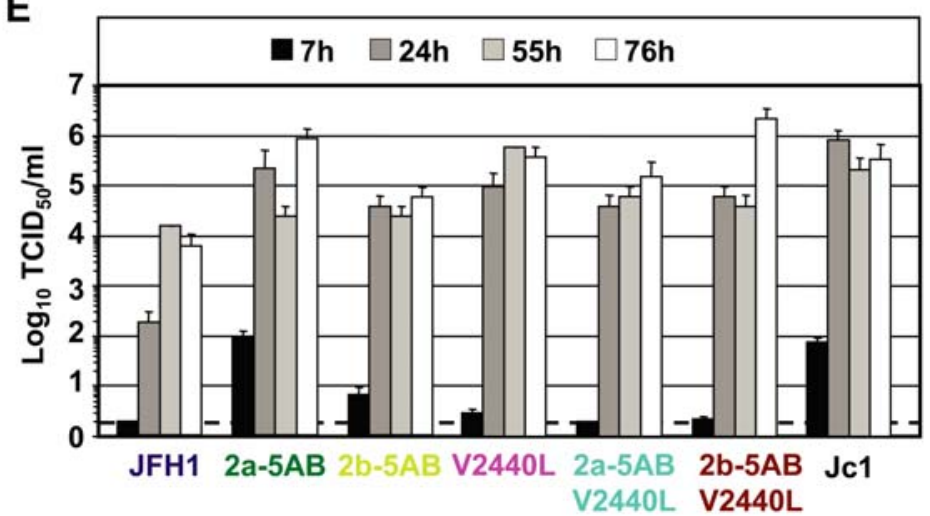

Figure 9. Properties of NS5A-B cleavage site mutants. (A) Alignment of amino acid consensus (con) sequences of various HCV genotypes or individual HCV isolates ( $\mathrm{H} 77, \mathrm{Con} 1, \mathrm{JFH} 1$ ) at the P-side of the NS5A-B cleavage site. JFH1 (upper lane) was used as master sequence. Numbers at the top refer to the P-side positions. Amino acid sequences of cleavage site mutants are given in the lower part of this panel (CONSTRUCTS). (B) Time course of replication of subgenomic sgNS3/JFH1-Luc replicons specified in the top. Only the early time points up to $24 \mathrm{~h}$ post infection are shown. (C) Dose-response assay of cleavage site mutants. Subgenomic replicons were transfected into Huh7-Lunet cells and treated $4 \mathrm{~h}$ later with escalating concentrations of DEBIO-025. After $72 \mathrm{~h}$ cells were lysed and luciferase activity was determined. Values are normalized to transfected control cells that were mock treated. The means and standard deviations of two independent experiments are shown in panel B and C. (D) Replication kinetic of cleavage site mutants in Huh7-Lunet cells with a stable knock-down of CypA. Cells transfected with constructs specified in the top were lysed at given time points and luciferase activity was determined. Values were normalized for transfection efficiency as determined with the $4 \mathrm{~h}$ value. (E) Impact of NS5A-B cleavage site mutants on release of infectivity. Genomes depicted at the bottom of the panel were transfected into Huh7-Lunet cells and release of infectious HCV particles into the supernatant was determined by $\mathrm{TCID}_{50}$ assay at time points specified in the top. (D-E) The background of the assay is indicated with the horizontal dashed line. Means and standard deviations of a representative experiment are shown. doi:10.1371/journal.ppat.1000546.g009

compared to CyPA thus increasing the likelihood of the latter to interact with the viral replicase [26].

In an attempt to study the mode-of-action of CypA, we wanted to exploit the possibility to interfere with this protein pharmacologically by using DEBIO-025, a compound that shows promise in clinical trials for the treatment of chronic hepatitis C [42]. The advantage of this compound is its higher anti-HCV activity as compared to CsA and reduced immunosuppressive activity, the latter being due to reduced calcineurin affinity $[5,46]$. As shown here, dose-response assays with cell lines expressing various levels of CypA confirm that DEBIO-025 indeed targets this host cell factor: $\mathrm{HCV}$ replication can be sensitized to the antiviral activity of DEBIO-025 by stable knock-down of CypA whereas overexpression of CypA leads to partial DEBIO-025 resistance. Although these results confirm CypA as a DEBIO-025 target, it is not known whether other Cyps are also affected by this compound, because interference with these proteins (e.g. CypB) has no effect on $\mathrm{HCV}$ replication, which was used as a read-out in our assays.
By using replicons to select for DEBIO-025 resistance we identified Y1421F (Y391F in NS3) that resides in the NS3 helicase domain, two mutations in domain 2 of NS5A (D2229G/D253G; L2266F/L290F) and one mutation at the P3 cleavage site position of the NS5A-B junction (V2440A/V464A). Among these mutations, only V2440A (V464A in NS5A) reduced DEBIO-025 sensitivity and rendered HCV replication less dependent on CypA. We had recently identified a mutation at the very same site that enhances virus assembly about 100-fold without affecting RNA replication (V2440L/V464L) [40]. As shown here this mutation also confers DEBIO-025 resistance, renders HCV replication less dependent on CypA and slows down processing at the NS5A-B site. Results obtained with additional mutations at the NS5A-B site revealed a correlation between processing kinetics and CypA dependence (Table 2): mutants with slower cleavage kinetic require lower level of CypA and are less sensitive to DEBIO-025 treatment compared to wild type whereas mutants with accelerated cleavage kinetic are more dependent on CypA and more 
Table 2. Summary of phenotypes of NS5A mutants.

\begin{tabular}{lllllc}
\hline Mutant & Kinetic $^{1}$ & DEBIO-025 resistance & CypA dependence & Replication & Assembly $^{2}$ \\
\hline V2440A & $\downarrow \downarrow$ & $\uparrow$ & $\downarrow$ & $\downarrow$ & n.d. \\
V2440L & $\downarrow$ & $\uparrow$ & $\downarrow$ & $\uparrow . a$. & $\uparrow$ \\
$2 \mathrm{a}-5 \mathrm{AB}$ & $\uparrow$ & $\downarrow$ & $\uparrow$ & u.a. & $\uparrow$ \\
$2 \mathrm{~b}-5 \mathrm{AB}$ & $\uparrow$ & $\downarrow$ & $\downarrow$ & u.a. & $\uparrow$ \\
$2 \mathrm{a}-5 \mathrm{AB}$ V2440L & $\downarrow$ & $\uparrow$ & $\downarrow$ & $\uparrow$. \\
$2 \mathrm{~b}-5 \mathrm{AB}$ V2440L & $\downarrow$ & $\uparrow$ & $\downarrow$ & u.a. & $\uparrow$ \\
\hline
\end{tabular}

${ }^{1}$ Kinetic of cleavage at the NS5A-B site.

${ }^{2}$ Production of infectious virus as deduced from $\mathrm{TCID}_{50}$ assay.

$\downarrow$, lower than wild type.

$\uparrow$, higher than wild type.

n.d., not determined.

u.a., unaffected.

doi:10.1371/journal.ppat.1000546.t002

sensitive to DEBIO-025 treatment. So far we can only speculate about the underlying mechanism, but for any model the following observations should be considered:

1. CypA and CypB are PPIases which appear to bind to the Cterminal region of NS5B [24,26];

2. CypA and/or CypB may induce a conformational change in NS5B by acting on a critical proline residue in the C-terminal region of NS5B [24];

3. Based on the X-ray crystal structure of NS5B, the Cyp binding site appears to be poorly accessible for protein-protein interaction in the folded NS5B molecule and may only be accessible in the unfolded protein, e.g. during protein synthesis (reviewed in [47]);

4. Cleavage at the NS5A-B site occurs rapidly and NS5A-B precursors are present in very low amounts $[17,18]$.

One possible explanation for our observations is that the CypA binding site in the C-terminal region of NS5B might be accessible only in an extended (non-folded) conformation right after protein synthesis. Proper folding of NS5B may require liberation of the Nterminus from the polyprotein (i.e. cleavage at the NS5A-B site). NS5B folded in the absence of CypA may adopt a conformation that is enzymatically inactive or that is not competent to be incorporated into the replicase complex [48]. In this model binding of CypA to NS5B would be required to induce a conformation necessary for replicase formation or activity. Binding however, would be possible only during a short time period, i.e. prior to or shortly after release of full length NS5B from the polyprotein. In case of a delayed processing (V2440L/A) the time during which the CypA binding site is accessible is extended and therefore lower amounts of CypA would suffice to bind to NS5B. Nevertheless, also under these circumstances CypA is required for HCV replication thus explaining why these replicons are still susceptible to DEBIO-025, albeit to a lesser extent. In case of hyper-processing the time for CypA binding would be much shorter and therefore the chance for interaction with NS5B would be lower. This can however be compensated by higher CypA amounts. This model would also explain why the mutations modulating NS5A-B processing do not have an effect on RNA replication in naïve cells. Under normal conditions, CypA levels are not limiting and even in case of the hyper-processing mutants CypA amounts are sufficient to bind to NS5B. Only when CypA levels are reduced either by knock-down of CypA expression or by DEBIO-025 treatment the chance that CypA can bind to NS5B during the much shorter time period, compared to wild type, becomes apparent. In contrast, delaying processing will extend the time for CypA binding and therefore even lower CypA amounts would be sufficient for binding to NS5B. However, a too slow processing at the NS5A-B site appears to impair replicase activity as deduced from the reduced replication of the V2440A mutant.

The CsA resistance mutations residing in domain 2 of NS5A that have been described in a recent study [27] may affect NS5B activity indirectly by altering binding of NS5A to NS5B. It has been shown that NS5A binds to NS5B and this interaction appears to modulate $\mathrm{RdRp}$ activity [49]. Alternatively, these mutations may affect directly a function of NS5A required for RNA replication [50] or polyprotein cleavage kinetics, but these possibilities need to be addressed. Finally, the impact of altered cleavage kinetics on HCV assembly might be an epiphenomenon caused by the mutations in domain 3 of NS5A itself that is a major determinant of virus assembly $[15,16]$.

Another surprising observation we made is the higher sensitivity of replication of JFHl full length genomes towards CypA depletion and the impairment of virus production. This observation suggests that CypA may have an additional target outside of the minimal replicase. The most likely candidate is NS2, because it contributes indirectly to RNA replication and is required for assembly $[12,13]$ whereas all other proteins residing in the $\mathrm{N}$ terminal domain of the polyprotein (core, E1, E2, p7) are completely dispensable for RNA replication. In fact, the stronger inhibition observed with the JFH1 sgNS2-replicon containing NS2 and the impairment of HCV assembly supports this assumption. Since NS2 contributes to RNA replication indirectly by cleaving off itself from NS3, a possible explanation is that CypA supports proper folding of NS2. However, in addition to a viral factor CypA may also be required for host cell factor(s) contributing to virus production. In fact, preliminary results suggest that CypA may be required for integrity of lipid droplets (A. Kaul, C. Berger, and R. Bartenschlager, unpublished observation), which play a key role in HCV assembly [20-22]. Further studies will be required to identify CypA-dependent viral and host cell factors required for HCV RNA replication, assembly and eventually egress.

\section{Materials and Methods}

\section{Cell culture and cell lines}

All cell lines were grown in Dulbecco's modified minimal essential medium (DMEM; Life Technologies, Germany) supplemented with $2 \mathrm{mM}$ L-glutamine, nonessential amino acids, $100 \mathrm{U} /$ 
$\mathrm{ml}$ of penicillin, $100 \mu \mathrm{g} / \mathrm{ml}$ of streptomycin, and $10 \%$ fetal calf serum. The experiments were performed either in Huh7-Lunet cells supporting high level RNA replication or Huh7.5 cells that are highly infectable $[51,52]$. For selection of DEBIO-025 resistant JFH1 replicons Huh7-Lunet sgNS3/JFH1-neo/RFP replicon cells [39] were double-selected for 18 cell passages with $500 \mu \mathrm{g} / \mathrm{ml}$ of G418 and 0 or 0.5 or $1 \mu \mathrm{g} / \mathrm{ml}$ of DEBIO-025. For transient dose response experiments subgenomic Luc-JFH1 replicons were transfected into Huh7-Lunet cells and $4 \mathrm{~h}$ post electroporation cells were treated with various concentrations of Cyclosporine A (CsA) or DEBIO-025, respectively. Seventy two hours later the impact on replication was determined by luciferase assay.

\section{Plasmid construction}

All nucleotide and amino acid numbers refer to the JFH1 genome (GenBank accession no. AB047639) or the Conl genome (GenBank accession number AJ238799). The chimera Jc1-Luc was described recently [33]. For replication analyses the subgenomic reporter replicon $\mathrm{pFKI}_{389} \mathrm{Luc} / \mathrm{NS} 3-3^{\prime}{ }_{2} \mathrm{dg} J \mathrm{JFH}$ (abbreviated as sgNS3/JFH1-Luc) and the replication deficient mutant carrying a deletion of the NS5B active site (sg/luc/JFH1/ $\Delta \mathrm{GDD}$ ) were used [39]. The basic Conl construct pFK-rep PI-luc/ET has been described somewhere else [31]. To generate the subgenomic JFH1 replicon construct $\mathrm{pFKI}_{389} \mathrm{Luc} / \mathrm{NS} 2-3^{\prime}{ }_{\text {_dg_(designated sgNS2/ }}$ JFH1-Luc in this report) we performed two separate PCRs: the first one with sense primer S/EMCV190 (5'-AATGCAAGGT CTGTTGAATGT- $3^{\prime}$ ) and antisense primer A/EMCV-NS2 (5'CGTGCACAGGTGCGTCATACATGGTATCATCGTGTTT

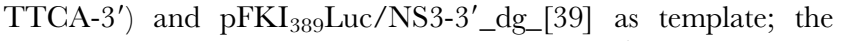
second one with primers A/JFH1/2894 (5'-TGACGGCGCACGCGATGCCAT-3') and S/EMCV-NS2 (5'-TGAAAA ACACGATGATACGATGTATGACGCACGTGTGCACG-3') and $\mathrm{pFKI}_{389}{ }_{2}$ Luc/Core-3'_dg_JFH [32] as template. Fragments were combined by overlap-PCR using S/EMCV190 and A/ JFH1/2894 and the resulting DNA fragment was inserted into $\mathrm{pFKI}_{389} \mathrm{Luc}_{\text {/Core-3' }}$ /DelE1E2_dg_JFH [32]. The final construct

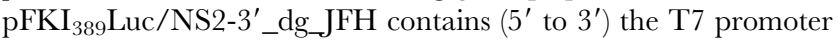
sequence fused to nucleotides 1 to 389 of the JFH1 consensus sequence, the firefly luciferase gene, the encephalomyocarditis virus (EMCV) IRES, the NS2 to 5B coding sequence, the 3' NTR of $\mathrm{JFH} 1$, the hepatitis delta virus genomic ribozyme (dg) and the T7 terminator sequence. Amino acid substitutions were introduced by PCR-based site-directed mutagenesis and amplified DNA fragments were analyzed by automated nucleotide sequencing using an ABI 310 sequencer (Applied Biosystems, Darmstadt, Germany).

\section{Generation of stable knock-down and rescue cell lines}

For CypA and CypB knock-down microRNA-based shRNA lentiviral vectors were produced by co-transfecting $293 \mathrm{~T}$ cells with transfer vectors encoding the puromycin resistance gene and a shRNA targeting CypA, CypB, or a control shRNA targeting luciferase, the HIV-1 packaging plasmid psPAX2, and a VSVg expression plasmid (pMD2.G) using Lipofectamine 2000 (Invitrogen). The shRNA targeting sequences were: luciferase, 5'tacaaacgctctcatcgacaag- $3^{\prime}$ (not present in the luciferase gene of the reporter replicon and reporter virus), CypA, 5'-ctggattgcagagttaagttta-3'; and CypB, 5'-gccgggtgatctttggtctctt- $3^{\prime}$. Transfection medium was changed the next day and viral supernatant was harvested $48 \mathrm{hrs}$ after transfection, clarified by centrifugation $(5 \mathrm{~min}$ at $200 \times \mathrm{g}$ ), and filtered through a $0.45 \mu \mathrm{m}$ syringe filter (Sarstedt). Huh7-Lunet and Huh7.5 cells were transduced on two consecutive days and placed into selection medium containing $5 \mu \mathrm{g} / \mathrm{ml}$ puromycin (Sigma) $72 \mathrm{~h}$ after transduction. For selected
Huh7-Lunet cell pools, knock-down of CypA and CypB was stable for at least 20 passages and cell growth as well as viability was not affected (long-term passage of Huh7.5 cell pools was not performed). For stable over-expression of CypA in CypA knockdown cells we used a lentiviral vector system. $293 \mathrm{~T}$ cells $\left(5 \times 10^{5}\right)$ were seeded in each well of a 6 -well cell culture plate in complete DMEM. About $24 \mathrm{~h}$ later, cells were transfected with lentiviral plasmids by using Lipofectamine 2000 or Lipofectamine LTX/ Plus Reagent (Life Technologies) according to the instructions of the manufacturer. To generate the CypA rescue cell lines, $2.5 \mu \mathrm{g}$ of the transfer vector pWPI-CypAwt or pWPI-CypA/H126Q was transfected together with $2 \mu \mathrm{g}$ of the packaging vector (pCMV) and $0.6 \mu \mathrm{g}$ of the VSV envelope vector (pMD.G) into 293T cells by lipofection as described above. After 16-24 h medium (2 ml) was replaced by fresh one and an additional $24 \mathrm{~h}$ later culture supernatant was filtered through $0.45 \mu \mathrm{M}$ pore size polyvinylidene difluoride (PVDF) syringe filter (Carl Roth GmbH, Karlsruhe Germany) and used for transduction. Huh7-Lunet/CypA knockdown cells were seeded at a density of $1-2 \times 10^{4}$ cells per well of a 12-well plate and inoculated with $0.5-1 \mathrm{ml}$ of filtered supernatant. Twenty four hours later, inoculation was repeated. Two to three days after transduction medium was replaced by fresh cell culture medium containing puromycin and/or blasticidin S. Drug concentrations were increased steadily during cell passages up to $10 \mu \mathrm{g} / \mathrm{ml}$ puromycin in case of Huh7-Lunet or $20 \mu \mathrm{g} / \mathrm{ml}$ in case of Huh7.5 and $20 \mu \mathrm{g} / \mathrm{ml}$ blasticidin $\mathrm{S}$ (Life Technologies).

\section{In vitro transcription and electroporation of HCV RNAs}

In vitro transcription and electroporation of HCV RNAs was performed as described previously [40]. Transfected cells were immediately diluted into complete DMEM and seeded as required for the given assay.

\section{Preparation of total RNA, amplification of replicon RNA by RT-PCR and cloning of amplified DNA fragments}

Total RNA was isolated from a confluent $6 \mathrm{~cm}$ diameter dish of Huh7-Lunet cells containing the sgJFH1-neo/RFP replicon by using the Nucleo Spin RNAII Kit (Macherey-Nagel, Düren, Germany) as recommended by the manufacturer. One $\mu \mathrm{g}$ total RNA and 50 pmol of primer A9482 (5'-GGA ACA GTT AGC TAT GGA GTG TAC C-3') were used for cDNA synthesis by using the Expand-RT system (Roche, Mannheim, Germany) as recommended by the manufacturer. Two to four microliters of the reaction mixture were used to amplify the complete open reading frame in two overlapping fragments with the Expand Long Template PCR kit (Roche) according to the instructions of the manufacturer. To amplify the $5^{\prime}$ half of the replicon, PCR was performed with primers S59-EcoRI (5'-TGT CTT CAC GCA GAA AGC GCC TAG-3') and A4614 (5'-CTG AGC TGG TAT TAT GGA GAC GTC C- $3^{\prime}$ ) and the PCR product was inserted into sgJFH1-Luc after restriction with EcoRI and SpeI. The 3' half of the HCV genome was amplified with primers S3813 (5'GGA CAA GCG GGG AGC ATT GCT CTC-3') and A9466MluI (5'-AGC TAT GGA GTG TAC CTA GTG TGT GCG-3') and after restriction with SpeI and MluI, the fragment was inserted into pFK-I ${ }_{389}$ neo/NS3-3'/Con [35]. Sequence analysis was performed with a set of primers covering the complete replicon sequence.

\section{Indirect immunofluorescence}

Huh7-Lunet and Huh7.5 derived cell lines were seeded onto glass cover slips in 24-well plates at a density of $2-4 \times 10^{4}$ cells per well. Three days after seeding cells were fixed by treatment with 
methanol at $-20^{\circ} \mathrm{C}$ for $10 \mathrm{~min}$., washed 3 times with PBS, incubated for $30 \mathrm{~min}$ in $5 \%$ normal goat serum or bovine serum albumin (diluted in PBS) and incubated for $1 \mathrm{~h}$ at room temperature (RT) with one of the following primary antibodies: antiCyPA rabbit polyclonal antiserum (BIOMOL International, LP) diluted 1:400, or antiCypB rabbit polyclonal antiserum (Affinity Bio Reagents) diluted 1:800, or antiNS5A mouse monoclonal antibody (9E10) [53]. After an extensive wash with PBS, cells were treated with Alexa Fluor 488 or 546 conjugated antibodies, targeting rabbit or mouse IgG domains (dilution $1: 1,000$ in normal goat serum) for $1 \mathrm{~h}$ at RT in complete darkness. Unbound secondary antibodies were removed by washing three times with PBS and once with water. DNA was stained with $4^{\prime}, 6^{\prime}-$ diamidino-2-phenylindole (DAPI; Molecular Probes, Karlsruhe, Germany) for $1 \mathrm{~min}$ at RT. Finally samples were mounted on slides with FluormountG (Southern Biotechnology Associates, Birmingham, USA) and analyzed by using fluorescence microscopy. For quantitation of immunofluorescence signals raw pictures were imported into the image $\mathrm{J}$ software package and pixel intensities based on 8-bit gray value were determined using the histogram function of the program (National Institutes of Health, Bethesda, MD, USA).

\section{Luciferase assays}

Quantification of luciferase reporter activity was used to determine transient HCV RNA replication as described previously [33]. In brief, transfected Huh7-Lunet cells were resuspended in $41 \mathrm{ml}$ complete DMEM and $1.5 \mathrm{ml}$ of the suspension was seeded in each well of a 12-well plate. For each time point, duplicates of wells were harvested. For dose response experiments, $4 \mathrm{~h}$ post electroporation transfected cells were treated in duplicate with different concentrations of cyclosporine A (CsA) or DEBIO-025 and analyzed $72 \mathrm{~h}$ later by luciferase assay. Cells were washed once with PBS, $350 \mu \mathrm{l}$ of lysis buffer $(0.1 \%$ Triton X-100, $25 \mathrm{mM}$ glycylglycine, $15 \mathrm{mM} \mathrm{MgSO}$, $4 \mathrm{mM}$ EGTA and $1 \mathrm{mM}$ DTT, $\mathrm{pH}$ 7.8) was added and freeze-thaw lysates were prepared. For each well, two times $100 \mu \mathrm{l}$ lysate was mixed with $360 \mu \mathrm{l}$ assay buffer (25 mM glycylglycine, $15 \mathrm{mM} \mathrm{MgSO}_{4}, 4 \mathrm{mM}$ EGTA, $1 \mathrm{mM}$ DTT, $2 \mathrm{mM}$ ATP and $15 \mathrm{mM} \mathrm{K}_{2} \mathrm{PO}_{4}$, pH 7.8) and, after addition of $200 \mu \mathrm{l}$ of a luciferin solution $(200 \mu \mathrm{M}$ luciferin, $25 \mathrm{mM}$ glycylglycine, $\mathrm{pH}$ 8.0), measured for $20 \mathrm{~s}$ in a luminometer (Lumat LB9507; Berthold, Freiburg, Germany). Kinetic of replication was determined by normalizing the relative light units (RLU) of the different time points to the respective $4 \mathrm{~h}$ value. For dose response experiments the RLU obtained with cyclosporine A (CsA) or DEBIO-025 treated cells were normalized to the corresponding values obtained with untreated cells.

\section{Metabolic radiolabeling of proteins and immunoprecipitation}

A total of $2.5 \times 10^{5}$ Huh7-Lunet/T7 cells [54] were seeded in each well of a 6 -well cell culture plate in complete DMEM. About $24 \mathrm{~h}$ later, cells were transfected with $2.5 \mu \mathrm{g}$ per well pTMNS33'JFH1 wild type or analogous constructs containing mutations specified in the results section or empty vector (pTM1-2) [55]. Transfection was performed by using Lipofectamine LTX/Plus Reagent (Life Technologies) according to the instructions of the manufacturer. After $4 \mathrm{~h}$, cells were washed once with methionine/ cysteine-free medium and starved for $1 \mathrm{~h}$. For radiolabeling cells were incubated for $90 \mathrm{~min}$ in $1 \mathrm{ml}$ methionine/cysteine-free medium, supplemented with $2 \mathrm{mM}$ glutamine, $10 \mathrm{mM}$ Hepes (pH 7.5), and $150 \mu \mathrm{Ci} / \mathrm{ml}$ of Express Protein labeling mix (Perkin Elmer, Boston). Cells were lysed either directly or washed with PBS and incubated in complete DMEM for 1 or $2 \mathrm{~h}$. Cell lysates were prepared by using NPB (50 mM Tris-Cl [pH 7.5], $150 \mathrm{mM}$ NaCl, 1\% Nonidet P-40, 1\% sodium deoxycholate, $0.1 \%$ SDS, $1 /$ $10,000 \mathrm{vol}$ aprotinin $(1 \mathrm{U} / \mathrm{ml}), 1 / 1,000 \mathrm{vol}$ leupeptin $(4 \mathrm{mg} / \mathrm{ml})$ and $1 / 100$ vol phenyl-methyl-sulfonyl-fluoride $(100 \mathrm{mM})$ ) and cleared by centrifugation at $13,000 \mathrm{~g}$ for $15 \mathrm{~min}$ at $4^{\circ} \mathrm{C}$. Cleared lysates were used for immunoprecipitation using either the NS5Aspecific monoclonal antibody 9E10 [53] or a polyclonal NS5Bspecific antibody. Immunocomplexes were dissolved in $70 \mu \mathrm{l} 2 \times$ sample buffer (400 mM Tris $\mathrm{pH} 8.8,10 \mathrm{mM}$ EDTA, $0.2 \%$ bromophenolblue, $20 \%$ sucrose, $3 \%$ SDS and $2 \%$ B-mercaptoethanol), separated in a $10 \%$ polyacrylamide-SDS gel and analyzed by autoradiography. $\mathrm{HCV}$-specific bands were quantified by phosphoimaging using the QuantityOne software (BioRad, Munich, Germany).

\section{Western blot analysis}

Huh7-Lunet cells of a confluent 6-well cell culture plate were washed once with ice-cold PBS and lysed with NP40 buffer buffer (50 mM Tris-HCl pH 8, $150 \mathrm{mM} \mathrm{NaCl,} \mathrm{1 \%} \mathrm{NP40).} \mathrm{After} 30 \mathrm{~min}$ incubation at $4^{\circ} \mathrm{C}$, cell debris was pelleted by centrifugation for $30 \mathrm{~min}$ at $10.000 \mathrm{~g}$ and at $4^{\circ} \mathrm{C}$. Four $\mu \mathrm{g}$ of cleared supernatant was diluted in $2 \times$ sample buffer, heated $5 \mathrm{~min}$ at $95^{\circ} \mathrm{C}$ and loaded onto a $12.5 \%$ polyacrylamide-SDS gel. After electrophoresis proteins were transferred to a PVDF membrane (PerkinElmer Life Sciences) for $1 \mathrm{~h}$ with an electric current of $1 \mathrm{~mA} / \mathrm{cm}^{2}$. Membrane was blocked in PBS supplemented with $0.5 \%$ Tween (PBS-T) and 5\% dried milk (PBS-TM) for at least $1 \mathrm{~h}$ prior to $1 \mathrm{~h}$ incubation with primary antibody diluted 1:1,000 in PBS-TM. Membrane was washed 3 times with PBS-T and incubated for $1 \mathrm{~h}$ with horseradish-peroxidase conjugated anti rabbit secondary antibody diluted 1:10,000 in PBS-TM. Bound antibodies were detected after 3 times washing with the ECL Plus Western Blotting Detection System (GE Healthcare Europe, Freiburg, Germany).

\section{Quantitation of HCV core protein}

$\mathrm{HCV}$ core protein in transfected cells or cell culture supernatants was quantified using the Ortho ${ }^{\circledR}$ trak-C $^{\mathrm{TM}}$ ELISA kit (Ortho Clinical Diagnostics, Neckargemünd, Germany). Lysates of transfected Huh7-Lunet cells were prepared by addition of $1 \mathrm{ml}$ per $6 \mathrm{~cm}$ diameter culture dish of PBS containing $1 \%$ Triton X-100, 1/10,000 vol aprotinin (1 U/ml), 1/1,000 vol leupeptin $(4 \mathrm{mg} / \mathrm{ml})$ and $1 / 100 \mathrm{vol}$ phenyl-methyl-sulfonylfluoride $(100 \mathrm{mM})$. Lysates were cleared by centrifugation $(18,000 \times \mathrm{g} ; 5 \mathrm{~min})$ and samples were diluted 1:10 or higher and processed for ELISA according to the manufacturer's protocol. Culture supernatants were filtered through $0.45 \mu \mathrm{m}$ pore-size filters and used directly for core ELISA. Colorimetric measurements were performed using a Sunrise colorimeter (Tecan Trading AG, Switzerland). Kinetic of replication was determined by normalizing the intracellular core amount of the different time points to the respective $4 \mathrm{~h}$ value. To determine the efficiency of core protein release, the percentage of extracellular core to total core protein (the sum of intra- and extracellular core protein) was calculated.

\section{Determination of virus titers in cell culture supernatants}

Virus titers were determined as described elsewhere with slight modifications [52]. Huh7.5 target cells were seeded at a concentration of $1.1 \times 10^{4}$ cells per well of a 96-well plate in a total volume of $200 \mu \mathrm{l}$ complete DMEM. Twenty four hours later, serial dilutions of virus containing supernatant were added with 6 wells per dilution. Three days later, cells were washed with PBS and fixed for $20 \mathrm{~min}$ with ice-cold methanol at $-20^{\circ} \mathrm{C}$. After three washes with PBS NS5A was detected with a 1:2,000 dilution of 
antibody $9 \mathrm{E} 10$ in PBS for $1 \mathrm{~h}$ at room temperature. Alternatively NS3 was detected with a 1:100 dilution of antibody 2E3 (kindly provided by H. Tang, Florida State University, USA) in PBS for $1 \mathrm{~h}$ at RT. Cells were washed again three times with PBS and bound primary antibodies were detected by incubation with peroxidase - conjugated or Alexa Fluor 546 - conjugated anti mouse antibody (Sigma - Aldrich), respectively, diluted 1:1,000 in PBS. After $1 \mathrm{~h}$ incubation at room temperature cells were washed three times with PBS and in case of peroxidase - conjugated antibody the Vector NovaRED substrate kit (Linaris Biologische Produkte GmbH, Wertheim, Germany) was used for detection. Virus titres $\left(50 \%\right.$ tissue culture infective dose per $\mathrm{ml}\left(\mathrm{TCID}_{50} / \mathrm{ml}\right)$ ) were calculated based on the method of Spearman and Kärber $[56,57]$.

\section{Supporting Information}

Figure S1 Impact of stable CypA or CypB knock-down on Dengue virus RNA replication. Huh7-Lunet cells (left panel) and Huh7.5 cells (right panel) either stably transduced with the shRNA vector (sh-control) or a vector encoding the CypA- or CypBspecific shRNA or naive cells were transfected with a subgenomic luciferase reporter replicon derived from the Dengue virus 2

\section{References}

1. Poynard T, Yuen MF, Ratziu V, Lai CL (2003) Viral hepatitis C. Lancet 362: 2095-2100.

2. Borel JF, Feurer C, Gubler HU, Stahelin H (1994) Biological effects of cyclosporin A: a new antilymphocytic agent. 1976. Agents Actions 43: 179-186.

3. Liu J, Farmer JD Jr, Lane WS, Friedman J, Weissman I, Schreiber SL (1991) Calcineurin is a common target of cyclophilin-cyclosporin A and FKBP-FK506 complexes. Cell 66: 807-815.

4. Handschumacher RE, Harding MW, Rice J, Drugge RJ, Speicher DW (1984) Cyclophilin: a specific cytosolic binding protein for cyclosporin A. Science 226 : 544-547.

5. Paeshuyse J, Kaul A, De Clercq E, Rosenwirth B, Dumont JM, Scalfaro P, Bartenschlager R, Neyts J (2006) The non-immunosuppressive cyclosporin DEBIO-025 is a potent inhibitor of hepatitis $\mathrm{C}$ virus replication in vitro. Hepatology 43: 761-770.

6. Ma S, Boerner JE, TiongYip C, Weidmann B, Ryder NS, Cooreman MP, Lin K (2006) NIM811, a cyclophilin inhibitor, exhibits potent in vitro activity against hepatitis $\mathrm{C}$ virus alone or in combination with alpha interferon. Antimicrob Agents Chemother 50: 2976-2982.

7. Bartenschlager R, Frese M, Pietschmann T (2004) Novel insights into hepatitis C virus replication and persistence. 63: 71-180.

8. Moradpour D, Penin F, Rice CM (2007) Replication of hepatitis C virus. Nat Rev Microbiol 5: 453-463.

9. Griffin SD, Beales LP, Clarke DS, Worsfold O, Evans SD, Jaeger J, Harris MP, Rowlands DJ (2003) The p7 protein of hepatitis C virus forms an ion channel that is blocked by the antiviral drug, Amantadine. FEBS Lett 535: 34-38.

10. Pavlovic D, Neville DC, Argaud O, Blumberg B, Dwek RA, Fischer WB, Zitzmann N (2003) The hepatitis C virus p7 protein forms an ion channel that is inhibited by long-alkyl-chain iminosugar derivatives. Proc Natl Acad Sci U S A 100: 6104-6108.

11. Premkumar A, Wilson L, Ewart GD, Gage PW (2004) Cation-selective ion channels formed by $\mathrm{p} 7$ of hepatitis $\mathrm{C}$ virus are blocked by hexamethylene amiloride. FEBS Lett 557: 99-103.

12. Jones CT, Murray CL, Eastman DK, Tassello J, Rice CM (2007) Hepatitis C virus $\mathrm{p} 7$ and NS2 proteins are essential for production of infectious virus. J Virol 81: 8374-8383.

13. Jirasko V, Montserret R, Appel N, Janvier A, Eustachi L, Brohm C, Steinmann E, Pietschmann T, Penin F, Bartenschlager R (2008) Structural and functional characterization of nonstructural protein 2 for its role in hepatitis C virus assembly. J Biol Chem 283: 28546-28562.

14. Huang L, Hwang J, Sharma SD, Hargittai MR, Chen Y, Arnold JJ, Raney KD, Cameron CE (2005) Hepatitis C virus non-structural protein 5A (NS5A) is a RNA-binding protein. J Biol Chem 280: 36417-36428.

15. Tellinghuisen TL, Foss KL, Treadaway J (2008) Regulation of hepatitis C virion production via phosphorylation of the NS5A protein. PLoS Pathog 4: e1000032. doi:10.1371/journal.ppat.1000032

16. Appel N, Zayas M, Miller S, Krijnse-Locker J, Schaller T, Friebe P, Kallis S, Engel U, Bartenschlager R (2008) Essential role of domain III of nonstructural protein 5A for hepatitis $\mathrm{C}$ virus infectious particle assembly. PLoS Pathog 4: e1000035. doi:10.1371/journal.ppat.1000035. isolate NGC (shown at the top). Cells were lysed at given time points after transfection, and luciferase activity in cell lysates was determined. Means and standard deviations of a representative experiment are shown. Details of this replicon will be described elsewhere. Cap, RNA cap structure; luc, firefly luciferase; ubi, ubiquitin.

Found at: doi:10.1371/journal.ppat.1000546.s001 (0.34 MB TIF)

\section{Acknowledgments}

We are grateful to U. Herian for excellent technical assistance. We thank Grégoire Vuagniaux (Debiopharma, Lausanne, Switzerland) for provision of DEBIO-025, C.M. Rice (Rockefeller University, New York, USA) for providing Huh7.5 cells and the NS5A monoclonal antibody 9E10, D. Trono (University Lausanne, Switzerland) for provision of the pWPI-based retroviral transduction system and A. Davidson (University of Bristol, U.K.) for gift of the Dengue virus NGC molecular clone.

\section{Author Contributions}

Conceived and designed the experiments: AK MZL VL RB. Performed the experiments: AK SS CB TP JS SK MZL. Analyzed the data: AK SS GB MZL VL JL RB. Contributed reagents/materials/analysis tools: TP JL. Wrote the paper: AK SS RB.

17. Bartenschlager R, Ahlborn LL, Mous J, Jacobsen H (1994) Kinetic and structural analyses of hepatitis $\mathrm{C}$ virus polyprotein processing. J Virol 68: 5045-5055.

18. Lin C, Pragai BM, Grakoui A, Xu J, Rice CM (1994) Hepatitis C virus NS3 serine proteinase: trans-cleavage requirements and processing kinetics. J Virol 68: 8147-8157.

19. Egger D, Wolk B, Gosert R, Bianchi L, Blum HE, Moradpour D, Bienz K (2002) Expression of hepatitis $\mathrm{C}$ virus proteins induces distinct membrane alterations including a candidate viral replication complex. J Virol 76: 5974-5984.

20. Boulant S, Targett-Adams P, McLauchlan J (2007) Disrupting the association of hepatitis $\mathrm{C}$ virus core protein with lipid droplets correlates with a loss in production of infectious virus. J Gen Virol 88: 2204-2213.

21. Miyanari Y, Atsuzawa K, Usuda N, Watashi K, Hishiki T, Zayas M, Bartenschlager R, Wakita T, Hijikata M, Shimotohno K (2007) The lipid droplet is an important organelle for hepatitis $\mathrm{C}$ virus production. Nat Cell Biol 9: 1089-1097.

22. Shavinskaya A, Boulant S, Penin F, McLauchlan J, Bartenschlager R (2007) The lipid droplet binding domain of hepatitis $\mathrm{C}$ virus core protein is a major determinant for efficient virus assembly. J Biol Chem 282: 37158-37169.

23. Wang P, Heitman J (2005) The cyclophilins. Genome Biol 6: 226.

24. Watashi K, Ishii N, Hijikata M, Inoue D, Murata T, Mivanari Y, Shimotohno K (2005) Cyclophilin B is a functional regulator of hepatitis C virus RNA polymerase. Mol Cell 19: 111-122.

25. Nakagawa M, Sakamoto N, Enomoto N, Tanabe Y, Kanazawa N, Kovama T, Kurosaki M, Maekawa S, Yamashiro T, Chen CH, Itsui Y, Kakinuma S, Watanabe M (2004) Specific inhibition of hepatitis C virus replication by cyclosporin A. Biochem Biophys Res Commun 313: 42-47.

26. Yang F, Robotham JM, Nelson HB, Irsigler A, Kenworthy R, Tang H (2008) Cyclophilin A is an essential cofactor for hepatitis $\mathrm{C}$ virus infection and the principal mediator of cyclosporine resistance in vitro. J Virol 82: 5269-5278.

27. Fernandes F, Poole DS, Hoover S, Middleton R, Andrei AC, Gerstner J, Striker R (2007) Sensitivity of hepatitis C virus to cyclosporine A depends on nonstructural proteins NS5A and NS5B. Hepatology 46: 1026-1033.

28. Heck JA, Meng X, Frick DN (2009) Cyclophilin B stimulates RNA synthesis by the HCV RNA dependent RNA polymerase. Biochem Pharmacol 77: 1173-1180.

29. Binder M, Kochs G, Bartenschlager R, Lohmann V (2007) Hepatitis C virus escape from the interferon regulatory factor 3 pathway by a passive and active evasion strategy. Hepatology 46: 1365-1374.

30. Binder M, Quinkert D, Bochkarova O, Klein R, Kezmic N, Bartenschlager R, Lohmann V (2007) Identification of determinants involved in initiation of hepatitis $\mathrm{C}$ virus RNA synthesis by using intergenotypic replicase chimeras. J Virol 81: 5270-5283.

31. Lohmann V, Hoffmann S, Herian U, Penin F, Bartenschlager R (2003) Viral and cellular determinants of hepatitis $\mathrm{C}$ virus RNA replication in cell culture. J Virol 77: 3007-3019.

32. Pietschmann T, Kaul A, Koutsoudakis G, Shavinskaya A, Kallis S, Steinmann E, Abid K, Negro F, Dreux M, Cosset FL, Bartenschlager R (2006) Construction and characterization of infectious intragenotypic and intergenotypic hepatitis $\mathrm{C}$ virus chimeras. Proc Natl Acad Sci U S A 103: 7408-7413. 
33. Koutsoudakis G, Kaul A, Steinmann E, Kallis S, Lohmann V, Pietschmann T, Bartenschlager $\mathrm{R}$ (2006) Characterization of the early steps of hepatitis $\mathrm{G}$ virus infection by using luciferase reporter viruses. J Virol 80: 5308-5320.

34. Welbourn S, Green R, Gamache I, Dandache S, Lohmann V, Bartenschlager R, Meerovitch K, Pause A (2005) Hepatitis C virus NS2/3 processing is required for NS3 stability and viral RNA replication. J Biol Chem 280: 29604-29611.

35. Lohmann V, Körner F, Koch JO, Herian U, Theilmann L, Bartenschlager R (1999) Replication of subgenomic hepatitis C virus RNAs in a hepatoma cell line. Science 285: 110-113.

36. Liu J, Chen CM, Walsh CT (1991) Human and Escherichia coli cyclophilins: sensitivity to inhibition by the immunosuppressant cyclosporin A correlates with a specific tryptophan residue. Biochemistry 30: 2306-2310.

37. Zydowsky LD, Etzkorn FA, Chang HY, Ferguson SB, Stolz LA, Ho SI, Walsh CT (1992) Active site mutants of human cyclophilin A separate peptidylprolyl isomerase activity from cyclosporin A binding and calcineurin inhibition. Protein Sci 1: 1092-1099.

38. Watashi K, Hijikata M, Hosaka M, Yamaji M, Shimotohno K (2003) Cyclosporin A suppresses replication of hepatitis $\mathrm{C}$ virus genome in cultured hepatocytes. Hepatology 38: 1282-1288.

39. Schaller T, Appel N, Koutsoudakis G, Kallis S, Lohmann V, Pietschmann T, Bartenschlager R (2007) Analysis of hepatitis C virus superinfection exclusion by using novel fluorochrome gene-tagged viral genomes. J Virol 81: 4591-4603.

40. Kaul A, Woerz I, Meuleman P, Leroux-Roels G, Bartenschlager R (2007) Cell culture adaptation of hepatitis $\mathrm{C}$ virus and in vivo viability of an adapted variant. J Virol 81: 13168-13179.

41. Combet C, Garnier N, Charavay C, Grando D, Crisan D, Lopez J, DehneGarcia A, Geourjon C, Bettler E, Hulo C, Le Mercier P, Bartenschlager R, Diepolder H, Moradpour D, Pawlotsky JM, Rice CM, Trepo C, Penin F, Deleage G (2007) euHCVdb: the European hepatitis C virus database. Nucleic Acids Res 35: D363-D366.

42. Flisiak R, Horban A, Gallay P, Bobardt M, Selvarajah S, Wiercinska-Drapalo A, Siwak E, Cielniak I, Higersberger J, Kierkus J, Aeschlimann C, Grosgurin P, Nicolas-Metral V, Dumont JM, Porchet H, Crabbe R, Scalfaro P (2008) The cyclophilin inhibitor Debio-025 shows potent anti-hepatitis $\mathrm{C}$ effect in patients coinfected with hepatitis $\mathrm{C}$ and human immunodeficiency virus. Hepatology 47: 817-826.

43. Gamble TR, Vajdos FF, Yoo S, Worthylake DK, Houseweart M, Sundquist WI, Hill CP (1996) Crystal structure of human cyclophilin A bound to the aminoterminal domain of HIV-1 capsid. Cell 87: 1285-1294.

44. Nakagawa M, Sakamoto N, Tanabe Y, Koyama T, Itsui Y, Takeda Y, Chen CH, Kakinuma S, Oooka S, Maekawa S, Enomoto N, Watanabe M (2005) Suppression of hepatitis C virus replication by cyclosporin a is mediated by blockade of cyclophilins. Gastroenterology 129: 1031-1041.
45. Chatterji U, Bobardt M, Selvarajah S, Yang F, Tang H, Sakamoto N, Vuagniaux G, Parkinson T, Gallay P (2009) The isomerase active site of cyclophilin a is critical for $\mathrm{HCV}$ replication. J Biol Chem [Epub ahead of print]

46. Ptak RG, Gallay PA, Jochmans D, Halestrap AP, Ruegg UT, Pallansch LA, Bobardt MD, de Bethune MP, Neyts J, De Clercq E, Dumont JM, Scalfaro P, Besseghir K, Wenger RM, Rosenwirth B (2008) Inhibition of human immunodeficiency virus type 1 replication in human cells by Debio-025, a novel cyclophilin binding agent. Antimicrob Agents Chemother 52: 1302-1317.

47. Appel N, Schaller T, Penin F, Bartenschlager R (2006) From structure to function: new insights into hepatitis $\mathrm{C}$ virus RNA replication. J Biol Chem 281: 9833-9836.

48. Liu Z, Yang F, Robotham JM, Tang H (2009) A Critical Role of Cyclophilin A and its Prolyl-Peptidyl Isomerase Activity in the Structure and Function of the HCV Replication Complex. J Virol [Epub ahead of print].

49. Shirota Y, Luo H, Qin W, Kaneko S, Yamashita T, Kobayashi K, Murakami S (2002) Hepatitis C virus (HCV) NS5A binds RNA-dependent RNA polymerase (RdRP) NS5B and modulates RNA-dependent RNA polymerase activity. J Biol Chem 277: 11149-11155.

50. Hanoulle X, Badillo A, Wieruszeski J-M, Verdegem D, Landrieu I, Bartenschlager R, Penin F, Lippens G (2009) Hepatitis C Virus NS5A protein is a substrate for the peptidyl-prolyl cis/trans isomerase activity of cyclophilins A and B. J Biol Chem epub ahead of print.

51. Blight KJ, McKeating JA, Rice CM (2002) Highly permissive cell lines for subgenomic and genomic hepatitis $\mathrm{C}$ virus RNA replication. J Virol 76: 13001-13014.

52. Friebe P, Boudet J, Simorre JP, Bartenschlager R (2005) Kissing-loop interaction in the $3^{\prime}$ end of the hepatitis $\mathrm{C}$ virus genome essential for RNA replication. J Virol 79: 380-392.

53. Lindenbach BD, Evans MJ, Syder AJ, Wolk B, Tellinghuisen TL, Liu CC, Maruyama T, Hynes RO, Burton DR, McKeating JA, Rice CM (2005) Complete replication of hepatitis C virus in cell culture. Science 309: 623-626.

54. Appel N, Pietschmann T, Bartenschlager R (2005) Mutational analysis of hepatitis $\mathrm{C}$ virus nonstructural protein $5 \mathrm{~A}$ : potential role of differential phosphorylation in RNA replication and identification of a genetically flexible domain. J Virol 79: 3187-3194.

55. Bartenschlager R, Lohmann V, Wilkinson T, Koch JO (1995) Complex formation between the NS3 serine-type proteinase of the hepatitis C virus and NS4A and its importance for polyprotein maturation. J Virol 69: 7519-7528.

56. Spearman C (1908) The method of "right and wrong cases" ("constant stimuli") without Gauss's formulae. Brit J Psychol 2: 227-242.

57. Kärber G (1931) Beitrag zur kollektiven Behandlung pharmakologischer Reihenversuche. Archiv für experimentelle Pathologie und Pharmakologie 162: 480-487. 